\title{
5. Exkurs: Frauen in der GTB
}

Die Gewerkschaft Textil-Bekleidung (GTB) war eine Frauengewerkschaft. Der Frauenanteil unter den Arbeitenden in der Textilindustrie schwankte zwischen 60 und 75 Prozent, in der Bekleidungsindustrie betrug er durchschnittlich 85 Prozent, so dass der Anteil der weiblichen Mitglieder in der GTB höher war als in jeder anderen Industriegewerkschaft. Nach Gründung der GTB im Jahr 1949 stieg der Frauenanteil in der Mitgliedschaft innerhalb eines Jahres von 52 Prozent auf 58 Prozent und sank in den folgenden zwanzig Jahren leicht, jedoch nie unter 53 Prozent. 1980 erreichte er wieder 58 Prozent und überschritt nach der deutschen Wiedervereinigung die 60-Prozent-Marke. ${ }^{1}$

Wie wirkte sich die Beschäftigten- und Mitgliederstruktur auf die Arbeit der GTB aus? Welche Rolle spielten Frauen in der betrieblichen Gewerkschaftsarbeit? Wie waren sie in den Entscheidungsgremien vertreten? Inwieweit machte die GTB Politik für Frauen? Wie ist das »Reich der Frauen«, die gewerkschaftliche Frauenarbeit, zu bewerten?

Diesen Fragen wird im Folgenden nachgegangen, wobei die gesellschaftlichen Rahmenbedingungen, insbesondere das den Frauen zugewiesene Rollenbild, von großer Bedeutung sind und in die Betrachtungen einfließen.

\subsection{Die Wegbereiterinnen im DTAV}

Bis 1908 war Frauen die Mitgliedschaft und Betätigung in politischen Vereinen in den meisten deutschen Gebieten verboten. Trotzdem bot die »Internationale Gewerksgenossenschaft der Manufaktur-, Fabrik- und Handarbeiter « die 1869 gegründet wurde und vor allem in Textilbetrieben aktiv war, als erste Organisation der Arbeiterbewegung Frauen die Mitgliedschaft an. 1885 gründeten Frauen in Berlin den »Fachverein der Mantelnäherinnen«, in dem sich die Näherin und So-

1 Alle Mitgliederzahlen stammen aus den schriftlichen Ceschäftsberichten des CTB-Hauptvorstandes zu den Gewerkschaftstagen. 
zialdemokratin Ottilie Baader stark engagierte. ${ }^{2}$ Im Jahr 1904 erhielt sie als erste Frau eine bezahlte Stelle beim SPD-Parteivorstand.

Erst nach Aufhebung der Sozialistengesetze im Jahr 1890 konnten sich Gewerkschaften freier organisieren, so dass 1891 der »Deutsche Textilarbeiter-Verband« (DTAV) gegründet wurde. Fünf weibliche Delegierte gehörten zu den Gründungsmitgliedern und 1904 wurde Martha Hoppe als erste Frau in die Zentralverwaltung des DTAV berufen. ${ }^{3}$ Beim bedeutenden Streik in der Crimmitschauer Textilindustrie 1903/1904 (siehe Kapitel 2.2.1) waren mehrere Frauen im Streikkomitee aktiv. Ein Jahr später wurde auch im Dachverband, der Generalkommission der Gewerkschaften Deutschlands, ein Frauensekretariat unter der Leitung von Ida Altmann gegründet.

Ab 1919 erzielten die Gewerkschaften deutliche Mitgliederzuwächse. Beim DTAV stellten Frauen dabei die Mehrheit der Mitglieder, beim 1920 gegründeten »Deutschen Bekleidungsarbeiter-Verband« betrug ihr Anteil zwei Drittel. ${ }^{4}$ Trotzdem wurden die speziellen Interessen berufstätiger Frauen in der männerdominierten Gewerkschaftsarbeit nicht ausreichend gewürdigt. Sprach eine Frau das Thema Hausarbeit und die Notwendigkeit der männlichen Mithilfe an, wurde das mit Heiterkeit aufgenommen und nicht ernstgenommen. Die geringe Repräsentanz von Frauen wurde in der DTAV-Mitgliederzeitung den Frauen selbst zugeschoben und gipfelte in der Frage:

»Ist die Gleichgültigkeit gegen alle Vorkommnisse des öffentlichen und rechtlichen Lebens, die leider weite Kreise der Arbeiterinnen noch immer beherrscht, nicht im höchsten Maße verantwortungslos? ${ }^{5}$

1926 versammelten sich 280 Gewerkschaftskolleginnen zum 1. Kongress der Textilarbeiterinnen Deutschlands in Gera. Der DTAV-Vorsitzende Karl Schrader appellierte an die Frauen, sich ihr Recht notfalls gegen die Männer zu erkämpfen, wobei er sich insbesondere auf die geringe Zahl weiblicher Delegierter bei Verbandskongressen bezog. ${ }^{6}$ Diese erste Textilgewerkschafts-Frauenkonferenz bot den Kolleginnen die Möglichkeit, ihre berufliche Situation sowie die Stellung innerhalb des Verbandes zu diskutieren und Forderungen zu entwickeln. Neben den Ansprüchen an Arbeitgeber und Regierung forderten die Frauen ein Organisationsleben, das sich besser auf die knapp bemessene Freizeit der Textilarbeite-

2 Vgl. CTB (1991): textil-bekleidung. 100 Jahre GTB. Sonderausgabe zum Jubiläum, S. 60.

3 CTB (1991): textil-bekleidung. 100 Jahre GTB. Sonderausgabe zum Jubiläum, S. 62.

4 CTB (1991): textil-bekleidung. 100 Jahre GTB. Sonderausgabe zum Jubiläum, S. 90 und 84.

5 Textil-Arbeiter, Ausgabe vom 13.2.1925, nach: GTB (1991): textil-bekleidung. 100 Jahre GTB. Sonderausgabe zum Jubiläum, S. 91.

6 CTB (1991): textil-bekleidung. 100 Jahre CTB. Sonderausgabe zum Jubiläum, S. 93. 
rinnen einstellt. Damit wurde ein zentrales Problem für die Beteiligung von Frauen thematisiert. Die in Gera versammelten Gewerkschafterinnen beanspruchten außerdem eine angemessenere Vertretung innerhalb des vergüteten Vorstandes, womit sie Erfolg hatten: Auf dem folgenden DTAV-Verbandstag 1927 wurde Else Niewiera in den hauptamtlichen Vorstand gewählt.

\subsection{An der Basis unverzichtbar, entbehrlich auf der Entscheidungsebene}

1949 nahmen am Gründungskongress der GTB 137 männliche und 23 weibliche Delegierte teil. ${ }^{7}$ In den ersten Hauptvorstand wurden neben sieben geschäftsführenden hauptamtlichen Mitgliedern auch sieben Ehrenamtliche gewählt. Da sich schon im DTAV ein Frauensekretariat etabliert hatte und nun zur neuen Gewerkschaft die noch stärker frauendominierte Bekleidungsherstellung hinzukam, galt Liesel Kipp-Kaule als für das Thema Frauenarbeit gesetztes GHV-Mitglied. Sie hatte bereits dem Vorstand für die britische Zone angehört und blieb die einzige Frau im Hauptvorstand; alle sieben ehrenamtlichen Mitglieder waren Männer. In den dreißigköpfigen Beirat, das höchste zwischen den Gewerkschaftskongressen tagende Beschlussgremium, wurden sieben weibliche Mitglieder gewählt, was einem Anteil von 23,3 Prozent entsprach (siehe Tabelle 3 im Anhang). Obwohl es auf dem GTB-Gründungskongress überwiegend um organisatorische Fragen ging, so ist doch bemerkenswert, dass bei der Gründung der "Frauengewerkschaft« keine einzige Frau das Wort ergriff.

Eine objektive Bewertung dieser Beobachtung ist jedoch ohne Blick auf die Gesellschaft der 1950er Jahre nicht möglich. Im Idealbild dieser Zeit war der Mann der Ernährer der Familie, die Frau hatte mit »ihrer Verheiratung ihren eigentlichen Beruf gewählt, nämlich den der Gattin, Mutter und Hausfrau«, wie noch 1954 in der Mitgliederzeitung »textil-bekleidung « zu lesen war. ${ }^{8}$ Das traditionelle Bild von der natürlichen Mutterrolle der Frau war durch den Nationalsozialismus propagiert und verstärkt worden und wurde auch nach Ende der Naziherrschaft nicht grundsätzlich infrage gestellt. Daran änderte auch der gegen konservativen Widerstand hart erkämpfte Gleichheitsgrundsatz im Grundgesetz nichts, obwohl die Widersprüche auf der Hand lagen. In den 1950er Jahren stellten Frauen ein Drittel aller Erwerbstätigen und jeder vierte Industriearbeitsplatz war von einer

7 Zum Vergleich: Beim DCB-Cründungskongress 1949 waren unter den 487 Teilnehmenden nur 14 Frauen.

8 Pelke (1954): Zwischen Arbeit und Familie: Die berufstätige Frau. 
Frau besetzt. ${ }^{9}$ Viele Frauen waren unverheiratet oder verwitwet, weil ihre Männer nicht aus dem Krieg zurückgekehrt waren.

Auch die Berufstätigkeit der verheirateten Frau war keine Ausnahme. 1950 ergab eine GTB-Befragung, dass von den beschäftigten Frauen in der Textil- und der Bekleidungsindustrie 45 Prozent verheiratet waren. ${ }^{10}$ Im Jahr 1954 waren es sogar 62 Prozent und drei Viertel von ihnen hatten Kinder. ${ }^{11}$ Somit war die "Mitarbeit« von Frauen in vielen Familien eine Tatsache, in der Regel eine wirtschaftliche Notwendigkeit. Von einem Recht auf Arbeit, wirtschaftlicher Eigenständigkeit oder Verwirklichung von Frauen im Beruf war selbst bei Gewerkschaften nicht die Rede. Ihre biologische und damit auch gesellschaftliche Bestimmung blieb ihre Rolle als Mutter und damit auch als Betreuerin und Erzieherin. So sagte Maria Weber, Mitglied des Geschäftsführenden DGB-Bundesvorstandes, noch auf der Frauenkonferenz 1959:

»Wir sind der Meinung, daß die Betreuung der Kinder durch die Mutter für die Entwicklung der Kinder nicht hoch genug bewertet werden kann und daß die Kinder, selbst wenn sie in gut geleiteten Kindergärten oder Horten tagsüber sind, etwas entbehren. [...] Wir möchten die Mütter zu Hause bei ihren Kindern sehen. $\ll^{12}$

Trotz Gleichheitsartikel im Grundgesetz bedurfte die Erwerbstätigkeit von verheirateten Frauen der Zustimmung des Ehemanns, der sogar ohne Einverständnis der Ehefrau deren Arbeitsverhältnis kündigen konnte. Erst 1958 wurde die Gesetzeslage dahingehend geändert, dass Ehefrauen ein eigenes Bankkonto eröffnen konnten und Frauen mit ihrer Verheiratung nicht automatisch das Bestimmungsrecht über Einkommen und Vermögen dem Ehemann übertrugen. Sozialpolitisch wurde eine allenfalls temporäre Erwerbstätigkeit von Frauen durch das gültige Rentenrecht untermauert. Von 1957 bis 1967 konnten sich Frauen nach der Heirat ihre bereits eingezahlten Rentenversicherungsbeiträge auszahlen lassen und verloren damit ihren Anspruch auf eine eigenständige Alterssicherung. Dies manifestierte das Bild des Ehemanns als alleiniger Versorger, bei dessen Tod die Witwenrente reichen musste. ${ }^{13}$

Erst im Laufe der 1960er Jahre öffnete sich der gesellschaftliche Blick auf die Ausbildung und Berufstätigkeit von Frauen. Die Vollbeschäftigung war erreicht, Frauen wurden als Arbeitskräfte von der Wirtschaft umworben. Die GTB-Zeitung »textil-bekleidung« sah im Jahr 1964 in einer großen Frauenreportage den Wunschtraum der Frauenrechtlerinnen früherer Tage erfüllt, allerdings

9 textil-bekleidung, Ausgabe vom 14.7.1954, S. 3.

10 GTB: Geschäftsbericht 1949-1950 des Hauptvorstandes, S. 130.

11 GTB: Geschäftsbericht 1953-1954 des Hauptvorstandes, S. 79.

12 DGB: Protokoll der 3. Bundesfrauenkonferenz, 25.-27. Mai 1959 in Bremen, S. 45 und 47.

13 Deutsche Rentenversicherung (2009): Heiratserstattung. 
»mit Einschränkungen. Die Frau ist nicht mehr Untertan des Mannes, nicht mehr Leibeigene herrischen Willens, nicht mehr Spielball sexueller Wünsche, nicht mehr häusliches Arbeitstier. [...] Noch gibt es zu wenig Aufstiegsmöglichkeiten für Frauen, viel zu wenig Bildungschancen, noch werden sie von vielen Stellen ferngehalten. Manche althergebrachten Vorurteile sind noch nicht überwunden. Noch gibt es Leute, die bewusst oder unbewusst einer Entwicklung entgegentreten, die nicht aufzuhalten ist. Deshalb bleibt auch noch manches zu tun, bis die Frau auf allen Cebieten unserer Wirtschaft und Gesellschaft die volle Gleichberechtigung erlangt hat. Aber verhindern lässt sich das auf die Dauer nicht mehr. ${ }^{14}$

Erst in den 1970er Jahren wurde die Arbeit von Frauen nicht nur mit wirtschaftlicher Notwendigkeit begründet, sondern als Voraussetzung für ihre Emanzipation. ${ }^{15}$ Nun stellte die Frauenbewegung Ansprüche, die sozialliberale Koalition reformierte das Ehe- und Familienrecht. Im Bundesfamilienministerium wurde 1978 erstmals ein Arbeitsstab Frauenpolitik unter Leitung von Marlies Kutsch, ehemals Mitglied im Hauptvorstand der IG Bergbau und Energie, eingerichtet. Im Jahr darauf berief der Hamburger Senat Eva Rühmkorf zur ersten Gleichstellungsbeauftragten in Deutschland. ${ }^{16}$

Zwar hatte sich das gesellschaftliche Frauenbild verändert, dennoch blieb die Vorstellung von der besonderen Rolle der Frau als Mutter und in der Familie präsent. Angesichts der wieder gestiegenen Arbeitslosigkeit diskutierte die CSU noch in den 1980er Jahren, ob tatsächlich beide Ehepartner ein Recht auf Beschäftigung im Öffentlichen Dienst hätten. Die GTB-Spitzenfunktionärin Gerda Linde forderte daraufhin, den uralten Kreislauf von Mädchen ohne Ausbildung, die zu Frauen ohne Berufschancen und somit schlechter bezahlt und schneller entlassen werden, endlich zu durchbrechen. ${ }^{17}$

Das Engagement von Frauen in der Gewerkschaft fand somit in den ersten Nachkriegsjahrzehnten unter der Besonderheit statt, dass es sich eigentlich um eine Männerdomäne handelte. Die berufstätige Frau wurde trotz 7,9 Millionen erwerbstätiger Frauen als Ausnahme gesehen und dargestellt. Zudem mussten Frauen ihren gewerkschaftlichen Einsatz unter der Doppelbelastung von Beruf und Haushalt organisieren. Allein die beruflichen Belastungen waren aufgrund der oft harten Arbeitsbedingungen in der Textil- und der Bekleidungsindustrie erheblich. Die Arbeitgeber bauten darauf, dass ein Großteil der Frauen tatsächlich nur vorübergehend erwerbstätig war. So beklagte der GTB-Geschäftsbericht 1953/1954 den »Raubbau an der Gesundheit der Frauen«, die für einen 8-Stunden-

\footnotetext{
14 textil-bekleidung, Ausgabe 5/1964, S. 11 (»Frauen stehen ihren Mann«).

15 So Menschik (1974): Gleichberechtigung oder Emanzipation?, S. 123.

16 Vgl. textil-bekleidung, Ausgabe 7/1978, S. 20

17 Linde (1984): Wachsender Druck auf die erwerbstätige Frau, S. 14.
} 
Arbeitstag zuzüglich Pausen und Arbeitsweg aufwenden mussten, um dann in der »wenigen Freizeit« die »erheblichen häuslichen Verpflichtungen« zu erledigten. ${ }^{18}$ Auf dem Gewerkschaftstag 1953 äußerte sich ein Delegierter »erschrocken, [...] dass 26 bis 30 Prozent der Krankheitsfälle nervöse Erschöpfung sind «. ${ }^{19}$

Alltagsberichte von der ersten GTB-Frauenkonferenz 1957

»Wir haben große Bänder. Daran sitzen etwa 113 Menschen. [...] Nun ist es so, daß wir durch die monotone Arbeit am Fließband sowieso überbelastet werden, schon als Frau und Mutter und dann auch unsere jungen Mädchen. [...] Früher [wurden] Kinder an den Webstühlen geboren. Die Frauen kommen heute wohl herunter von den Fließbändern, aber wir haben die Frühgeburten. Die Frauen müssen nach Hause gefahren werden, und da kommt es vor, daß die Kinder im Auto geboren werden. Darum geht meine Bitte noch einmal dahin, irgendetwas zu unternehmen, damit diese Zustände geändert werden. ${ }^{20}$

Betriebsratsvorsitzende Hester Kratzer, Balisches Bekleidungswerk in Wilhelmshaven

»Ich fange morgens fünf Minuten vor 5 Uhr an. Dann läuft das Fließband [...] [und] wird nur bei Pausen abgestellt. Es läuft im gleichen Takt. Keinerlei Rücksicht wird auf das Ansteigen und auf das Absinken der körperlichen Leistungsfähigkeit genommen. [...] Bei Pausen [...] [kamen] Schächtelchen, Tröpfchen und alles mögliche zum Vorschein. Für den einen war es zum Aufputschen [...], für die andren zur Beruhigung, weil das Herz zu sehr flatterte. Deswegen fordern wir eine Verlängerung der Pausen. [...] Eine Näherin hat einen kürzeren oder leichteren Arbeitsprozess [...]. Die andere hat eine diffizilere Arbeit und muss länger daran arbeiten. Sie muss aber genau die gleiche Stückzahl machen wie die, die leichtere Arbeiten hat. [...] Nebenbei türmt sich etwas auf. Daß das eine seelische Belastung ist, werden die feststellen können, die am Fließband arbeiten. « $^{21}$ Betriebsrätin Anny Fischer, Mehler in Fulda

18 GTB: Geschäftsbericht 1953-1954 des Hauptvorstandes, S. 200.

19 GTB: Protokoll des 3. Ordentlichen Cewerkschaftstages, 5.-19. Juni 1953 in Düsseldorf, S. 106.

20 GTB: Protokoll der 1. Zentralen Frauenkonferenz, 28./29. September 1957 in Bielefeld, S. 40.

21 GTB: Protokoll der 1. Zentralen Frauenkonferenz, 28./29. September 1957 in Bielefeld, S. 66. 
Vor diesem Hintergrund kann man erahnen, welch einen Einsatz es für Frauen bedeutete, Funktionen in der betrieblichen und gewerkschaftlichen Interessenvertretung zu übernehmen, doch dies taten sie aller Widrigkeiten zum Trotz. Schon Anfang der 1950er Jahre hatten Frauen ein Drittel der Betriebsratsmandate inne. ${ }^{22} 1965$ wurde die Zahl der weiblichen Betriebsratsvorsitzenden erstmals veröffentlicht. $\mathrm{Zu}$ diesem Zeitpunkt wurde bereits über ein Viertel der Betriebsratsgremien von Frauen geleitet und 38 Prozent der Betriebsratsmitglieder waren weiblich. 1984 wählten die Beschäftigten dann zu 56 Prozent Frauen in die Betriebsräte, was ihrem Anteil an den GTB-Mitgliedern exakt entsprach, und 55 Prozent der Gremien wurden durch Frauen geleitet. $\mathrm{Zu}$ diesem Zeitpunkt betrug der Frauenanteil im Deutschen Bundestag gerade einmal 9,8 Prozent. ${ }^{23}$

Keine andere Gewerkschaft war bei den ehrenamtlichen Funktionen im Betrieb so stark von Frauen geprägt. 1978 gab es in den Textil- und Bekleidungsbetrieben 1.384 weibliche Betriebsratsvorsitzende - das waren 36,4 Prozent aller im DGB organisierten weiblichen Betriebsratsvorsitzenden, während der Mitgliederanteil der GTB innerhalb des DGB nur 3,8 Prozent ausmachte. ${ }^{24}$ Dabei ist zu berücksichtigen, dass die Kandidatur von Frauen für ein Betriebsratsmandat im Vergleich zu Männern durch die Mehrfachbelastung in Beruf und Haushalt sowie die Familienphasen erschwert war.

Weiter ist zu beachten, dass Frauen mehr Berufsunterbrechungen wegen Kindern, also kürzere oder durchbrochene Betriebszugehörigkeitszeiten zu verzeichnen hatten. Dies war nicht förderlich für eine Karriere als Betriebsrätin. Die höhere Fluktuation von Frauen erforderte mehr Anstrengungen in der Mitgliederwerbung. Fast zwei Drittel der Neumitglieder waren Frauen und diese wurden überwiegend durch Frauen von einer Mitgliedschaft überzeugt. Der Frauenanteil bei den Werbenden überschritt schon Anfang der 1970er Jahre die 50-ProzentMarke und schwankte ab Mitte der 1970er Jahre um die 60 Prozent. Die weiblichen Mitglieder waren also keinesfalls passiv, sondern vertraten die Beschäftigten im Betrieb und warben für die Gewerkschaft; ohne sie hätte kein erfolgreicher Streik geführt werden können. Für die organisatorische Stabilität waren sie von fundamentaler Bedeutung.

Um dem hohen Frauenanteil in der Mitgliedschaft bei gleichzeitiger Unterrepräsentanz in den Führungsgremien Rechnung zu tragen, wurde bereits auf dem Gewerkschaftstag 1951 die Satzung ergänzt: Bei der Wahl der ehrenamtli-

22 Alle Daten aus den Ceschäftsberichten des CTB-Hauptvorstandes.

23 Wikipedia-Artikel »Frauenanteil im Deutschen Bundestag seit 1949«, Tabelle 1 (Abruf am 11.2.2021).

24 Resch/Rummel (1986): Von allem die Hälfte, S. 86f. 
chen Hauptvorstandsmitglieder sei »die Gruppe der Frauen zu berücksichtigen «. ${ }^{25}$ Allerdings wurde schon hier eine Tendenz deutlich, die sich durch die gesamte GTB-Geschichte ziehen sollte: Männer mussten nicht etwa Frauen weichen, sondern die Gremien wurden um Frauen erweitert. So wurde 1951 die Anzahl der ehrenamtlichen Hauptvorstandsmitglieder um zwei erhöht, so dass mit Paula Müller aus Baden-Württemberg eine Frau ein Mandat übernehmen konnte (siehe Tabelle 2 im Anhang). ${ }^{26}$

Ab 1955 gehörten dem erneut erweiterten Hauptvorstand, der nun achtzehn Mitglieder hatte, drei Frauen an. Neben Liesel Kipp-Kaule, die dem Geschäftsführenden Hauptvorstand (GHV) angehörte, wurden zwei ehrenamtliche Kolleginnen nominiert. 1963 wurde Kipp-Kaule aus dem GHV abgewählt, so dass nur die beiden Ehrenamtlichen im Hauptvorstand blieben. 1965 wurde der ehrenamtliche Teil des Hauptvorstandes auf vierzehn Personen aufgestockt, von denen nun fünf Frauen waren. Allerdings wurden in den Folgejahren zwei ausscheidende Frauen durch Männer ersetzt, so dass der Frauenanteil im Hauptvorstand bis 1990 bei 23,8 Prozent stagnierte - dem gleichen Anteil wie 25 Jahre zuvor.

Auch bei den Delegierten für die Gewerkschaftstage gab es eine Kontinuität der Männerdominanz (siehe Tabelle 3 im Anhang). Der Frauenanteil an den Stimmberechtigten von 14,4 Prozent beim Gründungskongress 1949 stieg bis 1971 nur auf 15,8 Prozent. 1978 wurde die 30-Prozent-Marke fast erreicht, aber auf dem Gewerkschaftstag 1982 sank der Frauenanteil wieder auf 26,5 Prozent. 1986 wurde das Allzeithoch von 36,2 Prozent erreicht, das immer noch deutlich unter dem Frauenanteil in der Mitgliedschaft lag, der 54 Prozent betrug. Lediglich im Beirat verdoppelte sich der Frauenanteil in den Jahren ab 1986 auf über 40 Prozent.

Bei den 164 Hauptamtlichen mit politischen Aufgaben startete die GTB 1950 mit zehn Frauen, was einem Anteil von 6,1 Prozent entspricht. Eine Frau gehörte dem GHV an, drei von 95 Verwaltungsstellen wurden von einer Frau geleitet. Die Anzahl der politischen Funktionär:innen stieg bis 1960 auf 176, darunter nur noch sechs Frauen (siehe Tabelle 4 im Anhang). Zwei Frauen arbeiteten in der Hauptvorstandsverwaltung, zwei Frauen in Bezirksleitungen und eine Geschäftsführerin leitete eine Verwaltungsstelle. Der Frauenanteil war auf 3,4 Prozent gesunken und erreichte erst 1967 wieder die Marke von 1952. Erst in den 1980er Jahren überstieg er die 10-Prozent-Marke und betrug 1989 vor der Wiedervereinigung mit zwanzig politischen Funktionärinnen 11,9 Prozent.

25 GTB: Protokoll des 2. Ordentlichen Gewerkschaftstages, 30. Mai-2. Juni 1951 in Düsseldorf, S. 213. Laut GTB-Satzung wurde die weibliche Mehrheit der Mitglieder dadurch als Gruppe definiert, die ein Teilnahmerecht am entscheidenden Exekutivorgan erhielt.

26 GTB: Protokoll des 2. Ordentlichen Gewerkschaftstages, 30. Mai-2. Juni 1953 in Düsseldorf, S. 172 in Verbindung mit S. 213. 
Die gesamtdeutsche Gewerkschaftseinheit brachte dann einen sprunghaften Anstieg des Frauenanteils. 1993 hatte die GTB 44 Gewerkschaftssekretärinnen und 105 Gewerkschaftssekretäre, zwölf Frauen standen an der Spitze von Verwaltungsstellen, der GTB-Bezirk Sachsen wurde von einer Frau geleitet, der ersten Bezirksleiterin in der Geschichte der Gewerkschaft. Durch die deutlich stärkere Vertretung von Frauen in Ostdeutschland stieg auch der Frauenanteil in ehrenamtlichen Gremien, blieb jedoch in den Vorständen auf allen Ebenen deutlich unter dem Frauenanteil in der Mitgliedschaft. 1993 betrug der Frauenanteil in den Verwaltungsstellenvorständen 43 Prozent, in den Bezirksvorständen 38 Prozent und im Hauptvorstand 33 Prozent. ${ }^{27}$

In Westdeutschland war bis zur deutschen Einigung in der gewerkschaftlichen Königsdisziplin, der Tarifpolitik, keine einzige Frau hauptamtlich tätig. Weder gab es eine Bezirksleiterin noch eine politische Sekretärin in der Tarifabteilung des Hauptvorstandes. Für die Gremien der Tarifpolitik wurden die Frauenanteile erst spät ermittelt und in den Geschäftsberichten ausgewiesen: 1978 waren 22 Prozent der bezirklichen Tarifkommissionsmitglieder weiblich, ${ }^{28}$ bis 1992 erhöhte sich ihr Anteil nur langsam auf 31 Prozent. ${ }^{29}$

\subsection{Von behüteten "Defizitwesen« und selbstbewusst fordernden Frauen}

Während die Frauen bei der GTB-Gründung noch stumm blieben, ergriff Marta Sieger als erste weibliche Diskussionsrednerin auf dem DGB-Gründungskongress im Oktober 1949 das Wort: »Erlaubt, daß auch einmal eine Frau zu Ihnen spricht. [...] Wir Frauen sind ja noch nicht so weit, wie die wohl schon durch Generationen geschulten Männer.« Die Frauen müssten weiter wachsen und lernen, »auf daß alle Frauen eines Tages einmal selbständig entscheiden und neben den Männern gleichberechtigt stehen können. $^{30}$

Dieser Einwurf macht deutlich, dass sich auch Frauen selbst als die »Besonderen-Minderen-Anderen« sahen, wie es die Psychologin Christine Morgenroth fomuliert. ${ }^{31}$ Dem männlichen Zeitgeist entsprechend erwiderte der DGB-Vorsitzende Hans Böckler vermeintlich höflich, aber bestimmt, dass die Männer die Sachentscheider seien: »Galanterie [hat] zu schweigen. Hier gilt es, nach Zweck-

27 CTB: Ceschäftsbericht 1990-1993 des Hauptvorstandes, S. $186 \mathrm{f}$.

28 GTB: Geschäftsbericht 1974-1977 des Hauptvorstandes, S. 428.

29 GTB: Geschäftsbericht 1990-1993 des Hauptvorstandes, S. 187.

30 DGB: Protokoll des Gründungskongresses, 12.-14. Oktober 1949 in München, S. $235 \mathrm{ff}$.

31 Morgenroth (1996): Die engagierte Frau, S. 40. 
mäßigkeitsgründen $\mathrm{zu}$ entscheiden. ${ }^{32}$ Die weiblichen Delegierten hatten versucht, ihre Favoritin, die Sozialdemokratin und GTB-Frau Liesel Kipp-Kaule bei der Wahl in den DGB-Bundesvorstand durchzusetzen, doch die Mehrheit der Männer entschied sich aus »Zweckmäßigkeitsgründen« für Thea Harmuth, die als Christlich-Soziale und Frau zwei informelle Quoten in einer Person erfüllte.

Bei der GTB ergriff auf dem zweiten Gewerkschaftstag im Jahr 1951 Wanda Kranz aus Bielefeld als erste weibliche Delegierte das Wort. Neben ihr und einer weiteren Kollegin beteiligten sich siebzig Männer an der Aussprache; beide Frauen unterlagen mit ihren Anliegen in den Abstimmungen. Erst auf dem Gewerkschaftstag 1953 griffen Frauen vehementer in die Debatte zum Geschäftsbericht ein. Im folgenden Geschäftsbericht für die Jahre 1953 und 1954 widmen sich schließlich 7 von 225 Seiten den Themen berufstätiger Frauen. Als Erfolg wird betont, dass Frauen in allen Gremien vertreten seien, wobei die größte Mitarbeit "natürlich « in den Betriebsräten zu verzeichnen sei. ${ }^{33}$

Im mündlichen Geschäftsbericht des Hauptvorstandes auf dem Gewerkschaftstag 1955 streifte Werner Bock das Thema gewerkschaftliche Frauenarbeit nur kurz. Die bayerische Delegierte Kathi Schill kritisierte die knappen Ausführungen und stellte fest, dass man angesichts der Tatsache, dass 58 Prozent der Mitglieder Frauen sind, zu diesem Thema wesentlich mehr hätte sagen können. Sie bemängelte den geringen Stellenwert der Frauenerwerbsarbeit in den Verwaltungsstellen und entsprechend auch auf dem aktuellen Kongress:

»Mir kommt es so vor, als betrachteten viele unserer Kollegen die Delegation zu Kongressen, Bezirksveranstaltungen usw. in der Hauptsache als Domäne der Männer. Ich bin anderer Auffassung. [...] Ihr müsst sie [die Frauen] als tatsächlich gleichberechtigt ansehen, und nicht die Gleichberechtigung, nur weil sie Euch unbequem ist und praktisch noch nie Realität war, beiseiteschieben in dem Clauben, daß sie für Euch nachteilig ist und Ihr durch die Frauen bedroht wäret. [...] Ich kann mir nicht recht zusammenreimen, daß man auf Tagungen die Kolleginnen wegen ihrer Mitarbeit, ihrer Aktivität und meinetwegen noch wegen ihrem Charme - soweit sie welchen entwickeln - lobt, im übrigen aber auf ihre Mitarbeit keinen allzu großen Wert legt. ${ }^{34}$

In der Folge diskutierten fünf Kolleginnen prägnant und selbstbewusst den Stellenwert der Gewerkschaftsarbeit für die Frauen in der Branche. Frauen müssten systematisch stärker angesprochen, qualifiziert und beteiligt werden. Die GTB

32 DCB: Protokoll des Cründungskongresses, 12.-14. Oktober 1949 in München, S. 237.

33 CTB: Geschäftsbericht 1953-1954 des Hauptvorstandes, S. 201.

34 CTB: Protokoll des 4. Ordentlichen Cewerkschaftstages, 5.-8. Juli 1955 in Frankfurt am Main, S. $120 f f$. 
solle eine Politik für die Mehrheit ihrer Mitglieder vorantreiben, die auf Erleichterungen für berufstätige Frauen abziele, wie die Forcierung des Kampfes um die 40-Stunden-Woche und die Begrenzung der Leistungssteigerungen. Für die gewerkschaftliche Frauenarbeit müssten feste und verbindliche Strukturen geschaffen werden. Gefordert wurde eine verbindliche Richtlinie, wonach in jedem der acht Bezirke eine Frauensekretärin die Arbeit koordinieren solle.

1956 hielt der Geschäftsbericht fest, dass in 52 der rund 130 Verwaltungsstellen Frauenausschüsse tätig seien, ${ }^{35}$ vier Jahre später gab es zwar weniger Verwaltungsstellen, aber trotzdem schon 82 Frauenausschüsse. ${ }^{36}$ Als zuständiges GHV-Mitglied teilte Liesel Kipp-Kaule die Kritik am mangelnden Stellenwert der Frauenarbeit in vielen Verwaltungsstellen. Allerdings seien die Frauen selbst oft zu passiv - eine Aussage, die dem zu dieser Zeit verbreiteten Denkmuster entsprach. Der Hauptvorstand bemühe sich um die Einstellung von Frauen für eine hauptamtliche Tätigkeit, aber viele darauf Angesprochene würden aus familiären Gründen oder wegen der Befürchtung, den Anforderungen nicht zu genügen, ablehnen. Eine Änderung der Arbeitsstrukturen, um bessere Voraussetzungen für hauptamtliche Gewerkschaftssekretärinnen zu schaffen, kam damals niemandem in den Sinn. Kipp-Kaule war sich aber der strukturellen Benachteiligung durchaus bewusst und gab den Kollegen auf dem Gewerkschaftstag 1955 die Erkenntnis mit auf den Weg:

»Leider ist es so, daß nicht immer nur der gute Wille der Kollegin bei euch maßgebend ist, sondern die Kollegin muss 200 Prozent besser sein als der Kollege, der sich um diese Stelle bewirbt. ${ }^{37}$

Auch die erste GTB-Frauenkonferenz 1957 stellte die Forderung nach mehr Gewerkschaftssekretärinnen in den Vordergrund. Darüber hinaus wurde auch die mangelnde Bereitschaft von Frauen kritisiert, eine hauptamtliche Funktion zu übernehmen, wobei Beschränkungen durch familiäre Pflichten nur bei Frauen gesehen wurden. So wurde ernsthaft diskutiert, ob mit neu eingestellten politischen Funktionärinnen ein mehrjähriges Heiratsverbot vereinbart werden solle. Die Delegierte Hilde Westpfahl aus Wuppertal wandte ein:

„Wir brauchen kein Zölibat. Wir müssen erreichen, daß die Erziehung gleichberechtigt erfolgt, in Elternhaus, Schule, Ausbildung. Es darf keine Diskriminierung mehr geben. Wir müssen den Anspruch haben, den Beruf zu erlernen, der uns zu-

35 GTB: Geschäftsbericht 1955-1956 des Hauptvorstandes, S. 176.

36 GTB: Geschäftsbericht 1959-1960 des Hauptvorstandes, S. 258.

37 GTB: Protokoll des 4. Ordentlichen Gewerkschaftstages, 5.-8. Juli 1955 in Frankfurt am Main, S. 157. 
sagt. Wir brauchen soziale Einrichtungen, Kindergärten und Kinderkrippen. Eventuell auch Heime oder Heimstätten, in denen Kinder, während der Zeit da sie nicht zur Schule gehen untergebracht werden können. Wir dürfen bei uns Frauen nicht immer so sehr unsere Fehler und das Negative in den Vordergrund stellen. ${ }^{38}$

Der Zeit geschuldet, wurde selbst in einer Gewerkschaft, deren Mitglieder mehrheitlich Frauen waren, das vorherrschende Rollenbild bedient. So ist im GTB-Geschäftsbericht zum Gewerkschaftstag $1961 \mathrm{zu}$ lesen:

»Die Löhne der Familienväter sollten solche Höhe haben, daß keine Mutter mehr gezwungen oder versucht wird, um des Familienunterhalts willen erwerbstätig zu sein. $\ll^{39}$

Zugleich wurde betont, dass die Frauen mehr als ein Drittel der Erwerbspersonen stellen und die Wirtschaft ohne sie nicht mehr denkbar sei.

Die GTB-Gewerkschaftstage 1959 und 1961 standen stark im Zeichen von politischen Richtungskämpfen. Die wenigen weiblichen Delegierten beteiligten sich überproportional an den Diskussionen. Insbesondere wehrten sie sich gegen offen oder in Anspielungen vorgetragene Positionen, Frauen seien schwer zu gewinnen und aktivieren. Sie verwiesen auf die Rahmenbedingungen, die Rolle als Mutter, Hausfrau und Erwerbstätige sowie die Erfahrungen der Arbeitskämpfe, in denen die Frauen »ihren Mann gestanden« hätten. Wenn man das gesellschaftliche Frauenbild dieser Zeit in Betracht zieht, traten die Frauen auf den Gewerkschaftstagen durchaus selbstbewusst auf, wenn auch innerhalb des begrenzten Spielraums, der ihnen von der männlichen Mehrheit zugestanden wurde. Partizipationsansprüche wurden formuliert und 1955 wurde Liesel Kipp-Kaule erstmals die teilweise Leitung und sogar das Schlusswort des Gewerkschaftstages übertragen.

Aber es gab auch warnende Stimmen. Generell hatte man den Frauen vorgeworfen, sich zu wenig einzubringen - aber jetzt, da Ansprüche selbstbewusster formuliert wurden, warnte man vor Übersteigerung. So gab der DGB-Vorsitzende Ludwig Rosenberg der DGB-Frauenkonferenz 1965 mit auf den Weg:

»Eine an sich unnatürliche Situation kann auch nicht dadurch in eine natürliche verwandelt werden, daß aus dem an sich selbstverständlichen Anspruch der Gleichberechtigung eine Bevorzugung werden soll. ${ }^{40}$

38 GTB: Protokoll der 1. Zentralen Frauenkonferenz, 28./29. September 1957 in Bielefeld, S. 31.

39 GTB: Geschäftsbericht 1959-1960 des Hauptvorstandes, S. 253.

40 DGB: Protokoll der 5. Bundesfrauenkonferenz, 6.-8. Mai 1965 in Berlin, S. 47. 
Als seine Aussage auf Kritik stieß, ergänzte er:

»[...] wir können die Demokratie nicht dadurch aufheben, daß wir von vornherein lauter Sondernaturschutzparks einrichten für alle möglichen Gruppen und Minderheiten - entschuldigen Sie, wenn ich das in dieser komischen Form sage, es richtet sich nicht gegen die Frauen. ${ }^{41}$

Der Gewerkschaftstag 1963 der GTB erlebte eine Premiere: In das siebenköpfige Gewerkschaftstagspräsidium wurden vier Kolleginnen gewählt. Stolz berichtete die Mitgliederzeitung vom »von Frauen regierten Kongress «. ${ }^{42}$ Dieses Präsidium war das erste Gremium, in dem sich die Zusammensetzung der Mitgliedschaft abbildete. Allerdings wurde auf demselben Kongress Liesel Kipp-Kaule, die einzige Frau im GHV, Opfer der Richtungskämpfe innerhalb der Organisation (siehe Kapitel 5.2). Der Gewerkschaftstag war zwar kurzfristig geschockt, ansonsten blieb diese Niederlage aber ohne großen Nachhall. Die Abteilung Frauen wurde nun von Martin Lange als neuem GHV-Mitglied geleitet. In seinem ersten Geschäftsbericht betonte er, wie organisationsfreudig Arbeitnehmerinnen seien, denn 60 Prozent der neuen Mitglieder waren Frauen. ${ }^{43}$ In patriarchalischer Manier lobte Lange die "gute Mitarbeit der Kolleginnen« in der Gewerkschaft und betonte,

»daß Frauen trotz ihrer besonders großen Belastung durch Erwerbsarbeit und Hausfrauen- und Familienpflichten sich für die allgemeine Gewerkschaftsarbeit bereitfinden ${ }^{44}$

Auf dem nächsten Kongress im Jahr 1965 stellten Frauen keine Ansprüche. Die Tatsache, dass alle Führungspositionen von Männer besetzt waren, wurde mit keinem Wort erwähnt. Alle GHV-Mitglieder kandidierten erneut, Martin Lange, der »Frauenbeauftragte«, erhielt mit 80 Prozent die höchste Zustimmung unter allen GHV-Mitgliedern. Ein Zeitzeuge berichtet, die »Traditionalisten« hätten sich zurückhalten müssen, da ihre Unterstützung der Kampfkandidatur von Martin Lange 1963 zur Abwahl von Kipp-Kaule geführt habe (siehe Kapitel 3.2.4). So wurde das Problem der reinen Männerriege den vermeintlich höherwertigen Themen untergeordnet. ${ }^{45}$

41 DGB: Protokoll der 5. Bundesfrauenkonferenz, 6.-8. Mai 1965 in Berlin, S. 71.

42 textil-bekleidung, Ausgabe 10/1963, S. 7.

43 GTB: Geschäftsbericht 1963-1964 des Hauptvorstandes, S. 189.

44 GTB: Ceschäftsbericht 1963-1964 des Hauptvorstandes, S. 193.

45 Laut Interview der Verfasser:innen mit dem ehemaligen CHV-Mitglied Wolfgang Stender am 25. Juli 2017. 
Auch auf dem Gewerkschaftstag 1968 wurde die fehlende Vertretung der Frauen im GHV nicht thematisiert, sondern nur die geringe Anzahl der weiblichen Delegierten allgemein bedauert und erneut mit der Aufforderung verbunden, doch auch Frauen zu wählen. Dazu die Delegierte Lisa Frerk:

»Das haben wir in den vergangenen Jahren immer wieder zu hören bekommen. Geändert hat sich nichts. [...] Wir müssen doch anerkennen, daß es eine Kollegin schwer hat, sich aktiv einzusetzen. Aber bedauerlicherweise muß ich auch sagen, daß es ihr oft schwergemacht wird. Deshalb sollten wir doch versuchen, ein ausgewogenes Verhältnis herzustellen. [...] Wir sollten nicht so viel von Gleichberechtigung reden, sondern wir sollten versuchen, sie zu leben. ${ }^{46}$

Ein Jahr zuvor hatte die GTB-Frauenkonferenz die verstärkte Mitarbeit von Frauen in den GTB-Gremien gefordert, aber ohne konkrete Maßnahmen dafür festzulegen, was auch bisher nie geschehen war. Auf dem Gewerkschaftstag 1971 präsentierte dann Gerda Linde, die für den verstorbenen Martin Lange in den GHV nachgewählt worden war, das »Programm für die Arbeitnehmerin« der GTB. Als sie nach einer Reihe von Rednerinnen zu diesem Thema feststellte, dass bisher nur Frauen das Wort dazu ergriffen hätten, fühlten sich einige Männer zu Unrecht kritisiert: »Man« mache keine Politik für Männer, sondern für alle, wenn nicht sogar explizit für Frauen.

Das präsentierte Programm für Frauen umfasste nicht nur ein Paket an tarif- und gesellschaftspolitischen Forderungen, sondern gleich $\mathrm{zu}$ Beginn auch ein Kapitel »Stärkere Beteiligung von Kolleginnen an der allgemeinen Gewerkschaftsarbeit und in den Gremien der Organisation«. Hauptanliegen war die Ermutigung der weiblichen Mitglieder zur Mitarbeit und Verantwortungsübernahme; ein weiterer Schwerpunkt war die »intensive Aufklärung der Mitglieder und Funktionäre über die Notwendigkeit der Wahl von Kolleginnen in alle Gremien unserer Organisation «. ${ }^{47}$

Deutlich wie nie zuvor bekannte sich schließlich das 1978 vom Gewerkschaftstag beschlossene GTB-Programm zur Gleichberechtigung von Mann und Frau. Darin wird festgehalten, dass die Erwerbstätigkeit der Frau nicht nur eine wirtschaftliche Notwendigkeit sei, sondern auch der Persönlichkeitsentfaltung diene. Die Reformen zur Realisierung des Gleichheitsgrundsatzes im Grundgesetz müssten weiter vorangetrieben werden; erwerbstätige Frauen dürften nicht als

46 CTB: Protokoll des 10. Ordentlichen Cewerkschaftstages, 16.-20. September 1968 in Berlin, S. 132.

47 CTB: Protokoll des 11. Ordentlichen Gewerkschaftstages, 4.-8.Oktober 1971 in Dortmund, S. 306. 
»Puffer« des Arbeitsmarktes angesehen und in Krisenzeiten auf Hausarbeit und Kinderpflege reduziert werden: ${ }^{48}$

»Mann und Frau müssen die gleichen beruflichen Chancen haben und sich frei entscheiden können für eine Berufstätigkeit, die Aufgaben in der Familie oder eine gleichzeitige Beteiligung in beiden Bereichen. Dazu sind tiefverwurzelte Vorurteile abzubauen. Die traditionelle Verteilung der Ceschlechterrollen ist in Frage zu stellen. ${ }^{49}$

Diese deutliche Neupositionierung wurde durch die Forderung nach Rahmenbedingungen ergänzt, die die angestrebten Ziele ermöglichen oder zumindest erleichtern sollten. Zur innerorganisatorischen Vertretung der Frauen äußerte sich das Programm jedoch nicht. Erst im 1982 vorgelegten Geschäftsbericht heißt es, dass eine erfolgreiche Lösung der frauenspezifischen Probleme nur möglich sei,

»wenn die Kolleginnen ihre Interessen in den Entscheidungsgremien der Cewerkschaft Textil-Bekleidung selbst durch aktive Mitarbeit vertreten können«. ${ }^{50}$

Frauen seien jedoch nach wie vor unterrepräsentiert und Anstöße für die Verbesserung dieser Situation müssten von den Gremien der gewerkschaftlichen Frauenarbeit kommen. Der Hauptvorstand solle dort tätig werden, »wo sich konkrete Ansatzpunkte bieten.$^{51}$ Dies wiederholt der nachfolgende, 1986 vorgelegte Geschäftsbericht wortwörtlich, hält aber fest, dass künftig mit Frauenförderplänen Anstöße zur Verbesserung gegeben werden sollen. ${ }^{52}$

Es bleibt festzuhalten, dass sich Frauen nicht nur aktiv in der Interessenvertretung auf Betriebsebene beteiligten, sondern sich schon in den 1950 er und 1960er Jahren selbstbewusst auf Kongressen zu Wort meldeten - auch wenn manche Frauen ihre Rolle selbst relativierten oder sich dafür entschuldigten, dass sie Kritik übten. Damit trifft auf die GTB nicht zu, was Lydia Schambach-Hardtke der gewerkschaftlichen Frauenarbeit 2005 in »Gender und Gewerkschaften« attestierte, nämlich dass diese bis Mitte der 1960er Jahre von einer zunehmenden

48 GTB: Protokoll des 13. Ordentlichen Gewerkschaftstages, 1.-6. Oktober 1978 in Mannheim, S. $327 f f$.

49 CTB: Protokoll des 13. Ordentlichen Cewerkschaftstages, 1.-6. Oktober 1978 in Mannheim, S. 328.

50 GTB: Geschäftsbericht 1978-1981 des Hauptvorstandes, S. 378.

51 GTB: Geschäftsbericht 1978-1981 des Hauptvorstandes, S. 378.

52 GTB: Geschäftsbericht 1982-1985 des Hauptvorstandes, S. 384. 
Folgebereitschaft gegenüber der männlich geprägten Gewerkschaftspolitik gekennzeichnet war. ${ }^{53}$

Auf den Gewerkschaftstagen wurden Ansprüche angemeldet, wobei viele Rednerinnen ihrer Zeit voraus waren. Aufforderungen, mehr Frauen zu beteiligen, erhielten meist Beifall, hatten aber keine Konsequenzen. Allerdings thematisierten auch die Frauen die Strukturen zu wenig, die ihrem stärkeren Mitwirken in der Gewerkschaft entgegenstanden, allenfalls wurden Frauen aufgefordert, sich trotz ungünstiger Rahmenbedingungen gewerkschaftlich $\mathrm{zu}$ engagieren. Kaum beachtet wurde dabei die Tatsache, dass die gleichberechtigte Teilhabe von Frauen auch ein Problem der Männer war, und zwar von der häuslichen Arbeitsteilung beginnend bis hin zum persönlichen Verzicht. Fiel die Kritik von Frauen an ihrer mangelnden Berücksichtigung scharf aus, wurde das von der männlichen Mehrheit meist schweigend hingenommen und erst anschließend in Privatgesprächen auf den Gängen kommentiert - und auf weibliches Desinteresse an stärkerer Mitarbeit reduziert.

Erst in den 1980er Jahren folgte auf Klagen, Appelle und Forderungen die Formulierung konkreter Maßnahmen. Frauenerwerbstätigkeit und die Beteiligung von Frauen am politischen und gesellschaftlichen Leben waren selbstverständlicher geworden, so dass 1986 auch in der GTB Maßnahmen zur Frauenförderung beschlossen wurden. Auf dem Gewerkschaftstag 1986 formulierte Gerda Linde erstmals den klaren Anspruch auf gleichberechtigte Teilhabe:

»Frauen wollen in allen Entscheidungsgremien entsprechend ihrem Mitgliederanteil vertreten sein. Frauen wollen auch bei den hauptamtlichen Funktionären nicht länger nur als Minderheit zu sehen sein. ${ }^{54}$

Linde forderte eine gezielte und systematische Politik. Sie beließ es nicht bei der Bitte um Mitberücksichtigung und Mitarbeit von Frauen, sondern unterstrich ihren Selbstvertretungsanspruch mit konkreten Ansatzpunkten zur Verwirklichung: Die Strukturen müssten hinterfragt werden und dürften sich künftig nicht nur an männlichen Lebenssituationen orientieren. Man müsse Lösungen finden, damit auch Frauen mit Kindern hauptamtliche Funktionen ausüben könnten; mit den alten Strickmustern sei man nicht weit gekommen. In bisher nicht gekannter Deutlichkeit führte Linde 1986 aus:

53 Schambach-Hardtke (2005): Gender und Gewerkschaften, S. 56.

54 GTB: Protokoll des 15. Ordentlichen Gewerkschaftstages, 5.-9. Oktober 1986 in Aachen, S. 96. 
»Solange Frauen in den Entscheidungsgremien nicht gleichberechtigt sind, in denen die Politik konzipiert und letztlich entschieden wird, so lange haben wir nur eine halbe Demokratie. ${ }^{55}$

Da dies Gerda Lindes letzte Rede als GHV-Mitglied war, blieb die Umsetzung dieser deutlich formulierten Ansprüche anderen überlassen.

\subsection{Frauenförderpläne statt Quote}

Am Gewerkschaftstag 1986 blickte die GTB auf eine fast 40-jährige Geschichte zurück, in der die Frauen ihren Anteil an wichtigen Gremien kaum verbessern konnten. Deshalb setzte der Zentrale Frauenausschuss nun auf das Instrument der Frauenförderpläne. Der Hauptvorstand legte dem Gewerkschaftstag einen Antrag mit dem Ziel vor, die gleichberechtigte Beteiligung von Frauen auf allen hauptamtlichen Ebenen und in allen gewerkschaftlichen Beschlussgremien $\mathrm{zu}$ erreichen. Die Antragskommission schlug den Passus »entsprechend der Mitgliederstruktur « vor und betonte gleichzeitig, dass dieser nicht als Quote, sondern als Präzisierung des langfristig zu erreichenden Zieles zu verstehen sei.

In der folgenden Diskussion sprachen sich Serv Hennes, im GHV für Personal zuständig, und der Vorsitzende Berthold Keller entschieden dagegen aus. Beide betonten, dass sie sich vehement für eine stärkere Beteiligung von Frauen einsetzen wollten, aber eine Quote ablehnten. Hennes zeigte sich zwar überzeugt, dass eine Mehrheit von Kolleginnen allen Gremien der Gewerkschaftsarbeit zugutekomme, doch brauche diese gewaltige Umstrukturierung Zeit, denn »niemand von uns wünscht einen Verdrängungswettbewerb oder gar einen Geschlechterkampf ${ }^{56}{ }^{56}$ Keller wollte das Thema vorantreiben, "wie sich das manche nicht so vorstellen ${ }^{57}{ }^{57}$ allerdings sei das, was die Antragskommission vorschlage, eine Quotierung.

In der Diskussion auf dem Gewerkschaftstag fand die von der Antragskommission vorgeschlagene Präzisierung die Unterstützung von Frauen und Männern. Waltraud Hessedenz, neu gewähltes GHV-Mitglied, wehrte sich dagegen, dass das Wort »Quote« allein schon Panik auslöse. Eine proportionale Beteiligung sei in mancherlei Hinsicht Praxis, z. B. werde auch bei der Vertretung der Bereiche Textil und Bekleidung in den Gremien der jeweilige Mitgliederanteil herangezogen. ${ }^{58}$ Am Ende lehnte die Mehrheit der Delegierten die Präzisierung jedoch ab.

55 GTB: Protokoll des 15. Ordentlichen Gewerkschaftstages, 5.-9. Oktober 1986 in Aachen, S. 97.

56 GTB: Protokoll des 15. Ordentlichen Gewerkschaftstages, 5.-9. Oktober 1986 in Aachen, S. 335.

57 GTB: Protokoll des 15. Ordentlichen Gewerkschaftstages, 5.-9. Oktober 1986 in Aachen, S. 341.

58 GTB: Protokoll des 15. Ordentlichen Gewerkschaftstages, 5.-9. Oktober 1986 in Aachen, S. 339. 
Eineinhalb Jahre später wurden gleich vier Frauenförderpläne in einer gemeinsamen Sitzung des Zentralen Arbeitskreises für Frauenfragen und des Hauptvorstandes beschlossen. Die Pläne betrafen Gesellschaft, Betriebe, ehrenamtliche und hauptamtliche Teilhabe in der GTB; ihre Forderungen waren als Bausteine angelegt, die sich gegenseitig bedingen. Damit wollte man nicht nur die Frauen in der eigenen Organisation fördern, sondern auch die Verbesserung der Lebens- und Arbeitssituation von Frauen generell vorantreiben. Gesellschaftspolitisch wurden ein Umbau des Ehegattensplittings, die Einführung eines Elternurlaubs, verbesserte Bildungsangebote und gute Kinderbetreuungseinrichtungen gefordert. In den Betrieben sollten die Betriebsräte Frauenförderpläne vereinbaren, Frauenbeauftragte bestellen und auf die Chancengleichheit bei Qualifizierung, Aufstieg und Entgelt achten.

Innerorganisatorisch verpflichtete sich die GTB zur verstärkten Qualifizierung von Frauen und zur Beseitigung von Hemmnissen bei der Beteiligung. Dazu müsse die zeitliche und örtliche Lage von Schulungen, Veranstaltungen und Gremiensitzungen überprüft sowie das Angebot der Kinderbetreuung verstärkt werden. Alle GTB-Beschäftigten sollten Frauen auch für eine hauptamtliche Tätigkeit motivieren; bei gleicher Qualifikation sollten Frauen bevorzugt eingestellt werden. Zur Kontrolle sollte auf den regionalen Delegiertenzusammenkünften und dem Gewerkschaftstag regelmäßig über Maßnahmen und Frauenanteil in den Gremien berichtet werden. Die Konzeption war mittel- und langfristig angelegt, denn die Erhöhung des Frauenanteils wurde nicht auf dem Weg der Verdrängung, sondern durch Neubesetzung frei werdender Positionen angestrebt.

Als eine erste Bilanz der Wirksamkeit der Frauenförderpläne gezogen wurde, hatte sich die Welt entscheidend verändert. Der Gewerkschaftstag im November 1990 fand in einem wiedervereinigten Deutschland statt und die Vorbereitungen zur Bildung einer gesamtdeutschen Textil- und Bekleidungsgewerkschaft nahmen enorme Ressourcen in Anspruch. Dennoch legte Waltraud Hessedenz in ihrem mündlichen Geschäftsbericht den Schwerpunkt auf die Frauenförderung. Sie verwies auf das Engagement von Frauen in Betriebsräten und örtlichen Tarifkommissionen und forderte, deren Beteiligung auch auf den höheren Ebenen durchzusetzen. Außerdem bedauerte sie, dass der Anteil der weiblichen Delegierten auf diesem Kongress auf 34,3 Prozent und damit um zwei Prozentpunkte gegenüber dem letzten Gewerkschaftstag gesunken war:

»Frauenförderung, das war beim letzten Gewerkschaftstag der gemeinsame Wille. Lasst es uns jedoch nicht nur gemeinsam wollen, tun wir auch gemeinsam 
etwas dafür! Man kann mir, glaube ich, nicht böse sein, wenn ich manche Entwicklungen enttäuschend finde. $\aleph^{59}$

Trotz der vielen aktiven Frauen an der Basis hatte sich in den vergangenen Jahren nur wenig bewegt. Die Arbeitskreise für Frauenfragen auf allen drei Ebenen der Organisation waren zwar Impulsgeber für eine lebendige Gewerkschaftsarbeit und der Organisationsgrad der Frauen stieg kontinuierlich. Die gewerkschaftliche Frauenarbeit vor Ort arbeitete vielfach mit offenen Formen und verstärkter Kinderbetreuung, so dass Frauen überproportional an Seminaren teilnahmen. Im Bereich der Hauptamtlichen stagnierte der Frauenanteil jedoch, ebenso in den Verwaltungsstellen- und Bezirksvorständen. Die vollmundigen Versprechen, mit denen Keller und Hennes 1986 die Frauenquote abgelehnt hatten, waren leere Worte geblieben: Es gab nicht eine zusätzliche Gewerkschaftssekretärin und auch beim Hauptvorstand stagnierte der Frauenanteil bei 28,6 Prozent. Erst die Bildung der gesamtdeutschen GTB im Jahr 1991 erhöhte den Frauenanteil spürbar.

Die Wirtschaftskrise 1993/1994 setzte der Textil- und der Bekleidungsindustrie schwer zu und damit verlor auch die GTB viele Mitglieder und ihre finanzielle Stabilität. Die Organisation kämpfte um ihr Bestehen, weshalb die gleichberechtigte Teilhabe von Frauen lediglich eine Nebenrolle spielte. Gleichwohl geriet die Arbeit der Frauen nicht ins Hintertreffen. Die weiblichen Delegierten hielten sich bei der Diskussion nicht mehr zurück und stellten die Mehrheit der Diskutierenden, obwohl der Frauenanteil auf dem Gewerkschaftstag 1994 nur bei 34,5 Prozent lag. Viele Kolleginnen berichteten von ihrer Arbeit und der aktuellen Arbeitsmarktsituation, von ihren Erfolgen bei der Beteiligung weiblicher Beschäftigter, der Gewinnung von Mitgliedern und dem Kampf gegen Benachteiligungen von Frauen im Betrieb. Die innergewerkschaftliche Beteiligung an Gremien war allerdings kein Thema.

\subsection{Gewerkschaftliche Frauenarbeit - Stachel in der Organisation oder einfach nur "Spielwiese «?}

Während sie dort, wo Gewerkschaftspolitik gemacht werde, nur symbolisch vertreten seien, beginne das Reich der Frauen in den Gremien der Frauenarbeit, schrieb Claudia Pinl, Journalistin und vormalige Redakteurin der »Gewerkschaftlichen Monatshefte«, im Jahr 1977. ${ }^{60}$ Historisch gesehen ging es bei der Frauenarbeit seit Gründung des Frauensekretariats zu Beginn des 20. Jahrhunderts um

59 GTB: Protokoll des 16. Ordentlichen Gewerkschaftstages, 4.-9. November 1990 in Würzburg, S. 75.

60 Pinl (1977): Das Arbeitnehmerpatriarchat, S. 99. 
die Gewinnung von weiblichen Mitgliedern und um deren Bildung und Qualifikation. Damit sollten die Voraussetzungen für die »Besonderen-Minderen-Anderen" geschaffen werden, damit diese aktiv am gewerkschaftlichen, wirtschaftlichen und gesellschaftlichen Leben teilnehmen können - ein Leben, das vielfach von Männern gesetzt wurde und deren Lebensbiografie entsprach. ${ }^{61}$ Pinl stellte die Frage, ob »das eigene Reich der Frauen « nicht gerade eine Emanzipation in der Gewerkschaft verhindere.

Diese Diskussion war nicht neu. Von Anfang an forderten Frauen in der GTB immer wieder, die Probleme der Frauenerwerbsarbeit in der Gesamtorganisation $\mathrm{zu}$ thematisieren. Sie sprachen sich nicht gegen die Gremien der Frauenarbeit an sich aus, betonten aber die Notwendigkeit, Politik für Frauen als Aufgabe aller zu begreifen. Anfang der 1970er Jahre blieb es nicht mehr bei Worten: Die Frauenausschüsse der GTB wurden in Arbeitskreise für Frauenfragen umbenannt und ausdrücklich für die Mitarbeit von Männern geöffnet. Dies führte nicht dazu, dass die Frauen die Autonomie verloren, denn nur wenige Männer arbeiteten mit. Dass sich Männer mit Frauenfragen befassten, war nicht völlig neu, denn schon zuvor hatte es auf der bezirklichen und der Hauptvorstandsebene einige männliche hauptamtliche Betreuer für die Frauenarbeit in den Gremien gegeben. Arbeitskreise für Frauenfragen sollten in jeder Verwaltungsstelle eingerichtet werden. Es gab sie in jedem Bezirk und auf der zentralen Ebene das Frauengremium.

Es stellt sich die Frage nach dem Stellenwert dieser Gremien der Frauenarbeit in der GTB: Waren sie eine Art »Beschäftigungstherapie« für Gewerkschaftsfrauen oder hatte man ihnen einen eigenen "Sandkasten « eingerichtet, wo sie aktiv sein konnten, ohne die Kreise der Männer zu stören? Fest steht, dass sich die Frauen die systematische Frauenarbeit in den Gewerkschaften erstritten hatten, was von Männern durchaus kritisch begleitet wurde. Sowohl innerhalb der GTB als auch bei den DGB-Frauenkonferenzen warnten die Vorsitzenden vor einer »Absonderung« der Frauen. Die Frauen seien »uns« gleichberechtigt, sagt Werner Bock zwar 1951, aber auch gleichverpflichtet und somit ohne Sonderrechte. ${ }^{62}$

Die GTB-Frauenarbeit konzentrierte sich zunächst darauf, die Arbeitsbedingungen der Frauen in der Textil- und der Bekleidungsindustrie zu verbessern. Die Frauen forderten geschlechtsunabhängige Lohnsysteme und stritten gegen die Nachtarbeit für Frauen und den zunehmenden Leistungsdruck. Bei Letzterem sahen die Gremien der Frauenarbeit in der Verkürzung der Wochenarbeitszeit und in der Verlängerung des Urlaubs entscheidende Hebel zur Veränderung. Ein Schwerpunkt lag auf dem Mutterschutz: Schwangere Frauen mussten im Akkord arbeiten, für das Verbot des Hebens und Tragens schwerer Lasten musste erst gekämpft werden und Mutterschaftsurlaub wurde erstmals 1979 eingeführt.

61 Pinl (1977): Das Arbeitnehmerpatriarchat, S. 100.

62 GTB: Protokoll des 2. Ordentlichen Gewerkschaftstages, 30. Mai-2. Juni 1951 in Düsseldorf, S. 47. 
Natürlich war auch die Tarifpolitik als gewerkschaftliches Kerngebiet Thema der Frauenausschüsse. Die Klagen insbesondere wegen struktureller Benachteiligung in den Tarifverträgen bei der Bewertung »typischer" Frauentätigkeiten wiederholten sich. Zwar war die gleiche Bezahlung für gleiche und gleichwertige Tätigkeiten seit eh und je in der gewerkschaftlichen Programmatik verankert, doch die Aktivitäten zur Realisierung hielten sich in Grenzen. Dies machten Frauen schon früh an ihrer mangelnden Beteiligung in den entscheidenden Gremien fest. Bereits auf der ersten GTB-Frauenkonferenz 1957 klagte die Betriebsrätin Mathilde Elschenbroich die Beteiligung von Frauen in den Tarif-Verhandlungskommissionen ein:

»Es geht doch nicht, daß wir in den Tarifkommissionen unsere Forderungen einbringen, bei den Tarifverhandlungen dann aber nicht mehr dabei sind. $\aleph^{63}$

Die Konferenz beschloss, mehr hauptamtliche Frauen und die Vertretung von Frauen in allen Tarif- und Verhandlungskommissionen zu fordern.

Kritik gab es auch wegen der geringen Beteiligung in anderen Gremien. So begrüßte Theresia Seidel, eine der beiden Geschäftsführerinnen einer Verwaltungsstelle, auf der 5. Frauenkonferenz 1971 die Kritik an der Unterrepräsentanz von Frauen in Politik und Wirtschaft. Sie plädierte allerdings dafür, auch die Verhältnisse in der eigenen Organisation kritisch zu betrachten. Hier seien Fortschritte bei der besseren Vertretung ebenfalls nur mühevoll und langsam erreicht worden. Frauen, so Seidel, seien durchaus bereit, auch auf höheren Ebenen Verantwortung zu übernehmen, müssten aber viel mehr Energie darauf verwenden als Männer und würden vielfach abqualifiziert:

"Was wir brauchen, ist kein Minderheitenschutz, sondern Verständnis und Anerkennung für unsere Arbeit und unsere Leistungen auch durch unsere Kollegen. ${ }^{64}$

Neben den Arbeitsbedingungen für Frauen in den Betrieben beschäftigten sich die Frauengremien durchaus auch mit der Rolle der Frau in der Gewerkschaft, und dies ab den 1970er Jahren zunehmend kritisch. Die meisten Frauen, die Führungsfunktionen in der Organisation erreichen konnten, hatten ihre aktive Arbeit in den Frauenausschüssen begonnen, was auch auf den Gewerkschaftstagen festzustellen war. Frauen, die dort das Wort ergriffen, waren in den meisten Fällen in der Frauenarbeit aktive Funktionärinnen. Dies zeigt, dass sich Frauen in der Frauenarbeit sowohl politisch gebildet als auch in Fragen von Strategie und Tak-

63 GTB: Protokoll der 1. GTB-Frauenkonferenz, 28./29. September 1957 in Bielefeld, S. 46.

64 GTB: Protokoll der 5. GTB-Frauenkonferenz, 23./24. April 1971 in Essen, S. 48. 
tik bei politischen Debatten qualifiziert hatten. Vor allem die Frauengremien auf Verwaltungsstellenebene boten dafür einen geschützten Raum.

Von den Frauenausschüssen kamen Forderungen, sich intensiver um die Verbesserung der Rahmenbedingungen für Frauenerwerbsarbeit zu kümmern und gesellschaftspolitisch Stellung zu beziehen, auch damit die Gewerkschaft für Frauen attraktiver würde. Die Veränderungen der Arbeitswelt, stärkere Beteiligung, menschenorientierte Arbeitskonzepte und der Wandel der Beschäftigungsstruktur führten zu veränderten Ansprüchen der Beschäftigten; Partizipation, Offenheit, Glaubwürdigkeit und Diskursfähigkeit waren gefragt. Für solche Ansprüche standen die Gewerkschaften trotz ihrer Mitgliedernähe nicht per se.

Weitere Impulse für die Gesamtorganisation kamen durch neue Arbeitsformen hinzu, denn im Sinne der veränderten Ansprüche entwickelte die GTB-Frauenarbeit in den 1980er Jahren eine Vielzahl kreativer Formen. Beispielsweise wurden die Feiern zum Internationalen Frauentag am 8. März nicht mehr nach dem Schema »Begrüßung, Referat, Schlusswort« gestaltet. Der Frauentag, mit dem sich die Gewerkschaftsvorstände noch zu Beginn der 1970er Jahre schwertaten, wurde zum Anlass für originelle Veranstaltungsformen der Gewerkschaftsfrauen. Außerdem führte die GTB ab 1986 regelmäßig Frauenaktionsmonate durch. Hier wurden Frauenthemen nicht nur in trockenen Veranstaltungen behandelt, sondern viele Ehrenamtliche organisierten Straßenaktionen, Kabaretts, Sketche und fröhliche und bunte Veranstaltungen, die zu einer neuen Kultur der Gewerkschaftsarbeit führten.

Die dadurch erzielte Aufmerksamkeit trug nicht nur zur Öffentlichkeitswirkung, sondern auch zur Stärkung des Selbstbewusstseins der Frauenarbeit bei. Dies wiederum wirkte sich auf die gesamte Gewerkschaft aus und »lockere« Formen wurden übernommen. Anstatt dem Referat des Gewerkschaftsvorsitzenden zu lauschen, interviewten Kolleginnen ihn publikumswirksam auf Veranstaltungen. Sogar Bezirkskonferenzen wurden zu offenen Foren umgebaut, die dann von Kolleginnen aus der Frauenarbeit moderiert wurden.

Die Frauenausschüsse, später die Arbeitskreise für Frauenfragen, waren ein Aktivitätsmotor der GTB. In ihnen konnten Probleme der Frauenerwerbsarbeit und gesellschaftspolitische Fragen relativ autonom besprochen und analysiert sowie Forderungen entwickelt und in die Gesamtorganisation eingebracht werden. $\mathrm{Ob}$ es um die Benachteiligung im Rentenrecht, die Problematisierung des Ehegattensplittings, das Staatsangehörigkeitsrecht, die Straffreiheit von Schwangerschaftsabbrüchen oder das Ehe- und Familienrecht ging, die GTB äußerte ihre Meinung zu diesen Themen, lange bevor sie von anderen aufgegriffen wurden. Bereits in den 1950er Jahren beschloss die GTB die Forderung nach Ganztagsbetreuungseinrichtungen für Kinder. 1965 forderte der GTB-Gewerkschaftstag vom 
DGB ein »Aktionsprogramm für die Lösung der Probleme, die sich aus der Erwerbstätigkeit der Frau ergeben ${ }^{6}{ }^{65}$

Die Zentralen Frauenkonferenzen waren Großereignisse der Organisation; ihre Diskussionen und Beschlüsse waren weitgreifend und betrafen nicht nur das Arbeitsleben direkt. Die Frauenarbeit in der GTB war somit sicher mehr als eine "Spielwiese« oder ein Ventil für die mangelnde inhaltliche und personelle Beteiligung von Frauen. Dennoch wurde - insbesondere bei der Frage der Frauenlöhne - der Zusammenhang zwischen den offensichtlichen Defiziten und der Geschlechterzusammensetzung in Tarifkommissionen und Vorständen lange Zeit nicht genügend beachtet. Erst ab den 1980er Jahren wurden in den Frauengremien Strategien entwickelt, um nicht mehr nur beratend oder fordernd gegenüber den Beschlussgremien der Gesamtorganisation aufzutreten, sondern auch den gerechten Anteil bei der personellen Besetzung dieser Gremien zu verwirklichen.

\subsection{Frauenlöhne in der Frauenbranche}

Seit ihrer Gründung hat sich die GTB die Lohngleichheit von Frauen und Männern zum Ziel gesetzt. Zu Beginn der 1950er Jahre wiesen die Tarifverträge jeweils eigene Tabellen für Männer, Frauen und Jugendliche aus. Frauen erhielten für die gleichen Tätigkeiten bis zu 20 Prozent weniger Lohn als Männer. So betrug 1953 der Stundensatz für einen Facharbeiter der Gruppe VI in der Textilindustrie in Westfalen 119 Pfennig, für Frauen lag er in der gleichen Lohngruppe bei 100 Pfennig. ${ }^{66}$ Die Effektivlöhne wiesen im Jahr 1954 eine Differenz von 23 Prozent auf. ${ }^{67}$ Der Geschäftsbericht vermerkt:

»Es war trotz größter Bemühungen nicht gelungen, die Verwirklichung der Lohngleichheit für Männer und Frauen bei gleicher Arbeit zu erreichen. ${ }^{68}$

Allerdings war die Abschaffung der Frauenlohngruppen trotz vieler Arbeitskämpfe wie der großen Auseinandersetzung 1953 im Tarifgebiet Westfalen kein Streikziel. Bei der Begründung der Lohngleichheit ging es nicht nur um Gerechtigkeit für Frauen. So führte der Vorsitzende Werner Bock im Hinblick auf die Tarifarbeit für die Bekleidungsindustrie aus:

65 GTB: Protokoll des 9. Ordentlichen Gewerkschaftstages, 11.-15. Oktober 1965 in Stuttgart, S. 227.

66 GTB: Geschäftsbericht 1953-1954 des Hauptvorstandes, S. $106 \mathrm{f}$.

67 GTB: Geschäftsbericht 1953-1954 des Hauptvorstandes, S. 110.

68 GTB: Geschäftsbericht 1953-1954 des Hauptvorstandes, S. 77. 
»Ein weiterer Übelstand war in der unterschiedlichen Entlohnung von Frauen- und Männerarbeit gegeben. Er bot die Möglichkeit, an Stelle der höher entlohnten Männer Frauen zu beschäftigen. ${ }^{69}$

Noch bevor das Bundesarbeitsgericht Anfang 1955 geschlechtsspezifische Lohngruppen für rechtswidrig erklärte, konnte die GTB die Abschaffung der Frauenlohngruppen in der Bekleidungsindustrie durchsetzen. Ebenso wie in der Textilindustrie gelang es, die schlichte Umwidmung in Leichtlohngruppen abzuwehren, wodurch die Tarifsätze erheblich anstiegen (siehe Kapitel 3.1.4). Innerhalb von fünf Jahren verkleinerte sich die Differenz der Effektivverdienste von Arbeitern und Arbeiterinnen in der Textilindustrie jedoch nur um rund vier Prozentpunkte auf nun 20 Prozent, dann blieb diese Differenz vier Jahrzehnte lang nahezu unverändert (siehe Tabellen 6 und 7 im Anhang). Anlässlich des Gewerkschaftstages 1961 wurde festgestellt, dass die Lohngleichheit bei gleicher Tätigkeit in den Tarifverträgen nun zwar gegeben sei.

»Das bedeutet allerdings nicht, daß damit bereits unsere gewerkschaftliche Forderung nach Lohngleichheit für Männer und Frauen bei gleicher und gleichwertiger Arbeit verwirklicht wurde. [...] Die Cleichwertigkeit der Leistungen unserer Frauen im Produktionsablauf, wo sie zweifelsohne vorhanden ist, wird in vielen Fällen noch nicht anerkannt. $\ll^{70}$

Das war und ist sicher der Hauptgrund der Ungleichheit von Männer- und Frauenlöhnen. Selbst bei gleicher Tätigkeit dürfte es nicht immer die gleiche Bezahlung gegeben haben, weil Männer oft aus fadenscheinigen Gründen höher eingruppiert wurden. Auch wenn der Anteil der Männer an der Facharbeit bis in die 1970er Jahre hinein deutlich höher war als bei den Frauen, haben sich auch spätere Veränderungen nicht ausgleichend auf die Lohndifferenz ausgewirkt.

Anfang der 1950er Jahre lagen die Frauenlöhne in der Bekleidungsindustrie rund ein Drittel unter denen der Männer; damit war die Lohndifferenz deutlich größer als bei Textil. Bis Anfang der 1960er Jahre verringerte sie sich um sechs Prozentpunkte auf 26 Prozent und sank in den folgenden Jahrzehnten auf 19 Prozent im Jahr 1994 und 15 Prozent in 2005. Fünfzig Jahre hatte es gedauert, den Abstand wenigstens zu halbieren, was sich auch dadurch nicht schönreden lässt,

69 CTB: Protokoll des 4. Ordentlichen Gewerkschaftstages, 5.-8. Juli 1955 in Frankfurt am Main, S. 65.

70 GTB: Geschäftsbericht 1959-1960 des Hauptvorstandes, S. 266. 
dass sich die Situation in der Gesamtindustrie noch ungünstiger darstellt. ${ }^{71} 1957$ lagen die Stundenlöhne der Frauen hier um 37 Prozent unter denen der Männer. Bis 1980 hatte sich diese Lücke um zehn Prozentpunkte verringert, in den 1980er und 1990er Jahren sank sie nur um jeweils einen Prozentpunkt und stagnierte bis 2005 bei 25 Prozent (siehe Tabellen 6 und 7 im Anhang).

In der GTB war die Verdienstlücke ein Dauerthema, auch wenn sie oft nachrangig bearbeitet wurde. Allerdings forcierte die GTB in den 1970er Jahren ein neues Eingruppierungsschema für die Bekleidungsindustrie, das die typischen Frauentätigkeiten höher bewertete. Insbesondere gelang es, qualifizierte Nähtätigkeiten immer wieder höherzugruppieren. In der Textilindustrie, in deren Tätigkeitskatalogen sich noch heute diskriminierende Beispiele finden lassen, kochte das Thema in den 1990er Jahren hoch. Angesichts des Lohnabstands der Frauen, der immer noch 20 Prozent zu den Männerlöhnen betrug, wurde eine grundsätzliche Neubewertung aller Tätigkeiten in einem gemeinsamen Entgeltrahmentarifvertrag für die Textilindustrie gefordert. Ein solcher Tarifvertrag konnte 2006 aber nur für Ostdeutschland durchgesetzt werden.

Trotz aller Bemühungen um Lohnangleichung ist nicht leicht zu erklären, warum die »Frauengewerkschaft« die gleiche Bewertung von Tätigkeiten, die insbesondere von Frauen ausgeübt wurden, nie zum Schwerpunkt einer Tarifrunde gemacht hat. Im Hintergrund mag eine Rolle gespielt haben, dass Frauentätigkeiten in der Textil- und der Bekleidungsindustrie im Durchschnitt aller Branchen noch relativ gut bezahlt waren. Außerdem war das Engagement der Männer auch deshalb gering, weil sie nicht gegen die Interessen ihrer Geschlechtsgenossen handeln wollten. Schließlich hätte die Angleichung der Frauenlöhne eine Einschränkung künftiger Männerlöhne zur Folge gehabt, da der Verteilungsspielraum in Tarifbewegungen begrenzt ist und eine strukturelle Anhebung der Frauenlöhne zulasten der Erhöhung von Männerlöhnen gegangen wäre.

\subsection{Frauen in der männergeführten GTB}

Mit Blick auf die geringe Frauenbeteiligung in den Führungsgremien der »Frauengewerkschaft" GTB und auf die Einkommensdifferenz stellt sich die Frage, weshalb die Organisation trotzdem attraktiv für Frauen war. In der fast 50-jährigen Geschichte der GTB traten über eine Million Frauen in die Gewerkschaft ein; ihr gewerkschaftlicher Organisationsgrad war höher als in jeder anderen Branche. Während die Gewerkschaftsarbeit in den 1950er Jahren fast überall eine

71 Zum Verarbeitenden Gewerbe, auf das sich die folgenden Zahlen beziehen, zählt das Statistische Bundesamt insbesondere Metallerzeugung und bearbeitung, Chemie- und Pharmaindustrie, Fahrzeugbau, Maschinenbau und Möbelherstellung. 
Männerdomäne war, herrschte in der Textil- und der Bekleidungsindustrie schon zu dieser Zeit ein erstaunliches Maß an Gleichberechtigung. In den Betriebsräten hatten Frauen bereits mehr als ein Drittel der Mandate inne. Durch die ab den 1960er Jahren verstärkt durchgeführte Erschließung der Bekleidungsindustrie wurden noch mehr Frauen für die Gewerkschaftsarbeit gewonnen. Bereits 1965 hatten über ein Viertel aller von der GTB betreuten Betriebe eine weibliche Betriebsratsvorsitzende.

Mit den gewerkschaftlichen Frauenausschüssen schufen sich Frauen einen Raum, in dem sie autonom diskutieren und Forderungen entwickeln konnten. Damit saßen sie zwar nicht an den Schalthebeln der Macht, beeinflussten die Organisation aber durchaus. Trotz der Mehrfachbelastung durch Erwerbsarbeit und Haushalt nahmen sie in hohem Maße Bildungsangebote an. Durch die örtlichen Tarifkommissionen reichte die Mitgliederbeteiligung bei der Aufstellung von Forderungen und der Gestaltung der Tarifrunden bis in die kleineren Betriebe. Auch dadurch konnten Frauen die Entscheidungsfindung beeinflussen. Obwohl Frauen ihre Beteiligung an der Tarifpolitik in den ersten beiden Nachkriegsjahrzehnten oft als unbefriedigend empfanden und dies auch artikulierten, ist die Berücksichtigung besonderer Frauenanliegen im Vergleich zu anderen Industriegewerkschaften doch erkennbar.

Wegen der Doppelbelastung von Beruf und Familie wurden kürzere Arbeitszeiten gefordert. Beim Ringen um die Absenkung der Wochenarbeitszeit von 48 auf 40 Stunden zog die GTB selbst an der IG Metall vorbei, die ansonsten taktgebend in der Tarifpolitik war. Gleiches gilt für die Dauer des Erholungsurlaubs, wo die Tarifpolitik der GTB über Jahrzehnte führend war. Auch bei den Erleichterungen für Akkordarbeiterinnen setzte die GTB Meilensteine, indem sie Mindestzuschläge für die Erledigung persönlicher Bedürfnisse und Erholungspausen in Tarifverträgen festschrieb. Ebenso wurde den Bedürfnissen von Frauen durch die Begrenzung von Mehrarbeit und den Vorrang von Freizeitausgleich Rechnung getragen, lange bevor eine solche Politik zum gewerkschaftlichen Mainstream gehörte.

Die Unzufriedenheit im Hinblick auf die Lohndifferenz gegenüber Männern mag sich auch deshalb in Grenzen gehalten haben, weil die Frauenlöhne bei Textil und bei Bekleidung bis in die 1970er Jahre hinein auf der Höhe des gesamtindustriellen Durchschnitts oder leicht darüber lagen, während die Männer schlechter entlohnt wurden als in der Gesamtindustrie (siehe Tabellen 6 und 7 im Anhang).

In den Arbeitskreisen für Frauenfragen wurden ab den 1970er Jahren Ansprüche an Arbeit und Leben formuliert, die nicht nur tarifpolitisch gelöst werden konnten, sondern sich auch an die Gesellschaft als Ganze wandten. Allgemeinpolitische Positionen wurden nicht nur stärker als bei anderen Gewerkschaften üblich auf den Kongressen thematisiert, auch die GTB-Publikationen gaben ihnen 
breiten Raum. Hier fanden sich Frauen mit ihren Problemen wieder, was sicher zur Identifikation mit ihrer Gewerkschaft beitrug.

Von Anfang an haben Frauen die Bedingungen ihrer Arbeit durch ihre Mitarbeit in der GTB mitgestaltet, anstatt ihr Los als erwerbstätige Frau nur zu erdulden. Dieser emanzipatorische Beitrag erfolgte zu einer Zeit, als selbst viele Frauen die Frauenerwerbstätigkeit als Ausnahme und »hoffentlich« nur vorübergehende Lebensphase sahen. Die Frauen in den Betriebsräten hatten Vorbildfunktion, zeigten sie doch, dass Frauen reden, handeln und sich gegenüber Männern durchsetzen können.

Frauen festigten ihre Position in der GTB, indem sie andere Frauen als Mitglieder warben. Dies war der entscheidende Faktor für den außergewöhnlich hohen gewerkschaftlichen Organisationsgrad der Frauen in den Textil- und Bekleidungsbetrieben. Gesamtgesellschaftlich gesehen sind Frauen deutlich weniger gewerkschaftlich organisiert als Männer; diese Organisationslücke war bei der GTB signifikant kleiner als in anderen Wirtschaftsbereichen: Im Jahr 1990 waren in Textil- und Bekleidungsbetrieben mit Betriebsrat 52,7 Prozent der Männer und 47,3 Prozent der Frauen Gewerkschaftsmitglied. ${ }^{72}$ Die "Organisationslücke« betrug somit fünf Prozentpunkte, während sie im Bereich der IG Metall fünfzehn Prozentpunkte ausmachte. ${ }^{73}$

Angesichts der Beschäftigtenstruktur konnten Tarifauseinandersetzungen nur mit Beteiligung von Frauen erfolgreich geführt werden, doch waren Frauen in den 1950er Jahren eher solidarische Begleiterinnen als Streikführerinnen. Dies änderte sich ab 1961, als die GTB erstmals einen Flächenstreik in der Bekleidungsindustrie organisierte (siehe Kapitel 4.1.4). Seitdem standen in vielen Arbeitskämpfen Frauen in der ersten Reihe.

Dennoch wurde Frauen die gleichberechtigte Beteiligung in den Spitzengremien Gewerkschaftstag und Hauptvorstand versagt. Die Männer besetzten selbstverständlich die Positionen, was im Hinblick auf die Mandate zum Gewerkschaftstag auch immer wieder kritisiert wurde. Schon in den $1950 e r$ Jahren gab es darüber heftige Diskussionen, die aber keine konkreten Maßnahmen zur Folge hatten. ${ }^{74}$ Für den GHV wurde eine gleichberechtigte Beteiligung oder zumindest die stärkere Berücksichtigung von Frauen nicht einmal diskutiert. Die Herrschaft der Männer wurde in den 1950er Jahren als quasi natürlich geduldet und auch in späteren Jahren nicht infrage gestellt.

72 CTB: Geschäftsbericht 1990-1993 des Hauptvorstandes, S. 423.

73 IG Metall: Detaillierte Auswertung der Betriebsratswahlen 1990 (unveröffentlicht).

74 So beantragte Anni Dorscheid 1954 im Beirat, die Gewerkschaftstagsmandate aus den Bezirken Baden-Württemberg und Bayern nicht zu bestätigen, da in diesen Bezirken nur Männer als Delegierte gewählt worden waren; vgl. CTB: Protokoll der Beiratssitzung vom 16.-19. Juni 1954, AdsD 5/CTBA0301001. 
Gleichwohl wurde zu allen Zeiten die geringe Zahl der Gewerkschaftssekretärinnen kritisiert, die zugrundeliegenden Rahmenbedingungen jedoch erst ab den 1980er Jahren analysiert. Bis dahin war eine Gewerkschaftssekretärin mit kleineren Kindern kaum denkbar und die große Ausnahme. Viele Frauen konnten sich eine hauptamtliche Tätigkeit wegen der damit verbundenen zeitlichen Belastung nicht vorstellen und zweifelten an ihrer entsprechenden Qualifikation, was teilweise auch für die höheren ehrenamtlichen Positionen galt. Trotzdem fühlte sich die große Mehrheit der Frauen in der Gewerkschaft gut vertreten. Dazu trug die oft familiäre Atmosphäre in der Mitgliedschaft bei, die auf den engen textilen Bereich beschränkt war. Außerdem führte die große Zahl der ehrenamtlich engagierten Frauen in den Verwaltungsstellen zu einem grundsätzlich respektvollen Umgang der Männer gegenüber Frauen, und zwar bis in die Führungsspitze hinein.

Die aufkommende Frauenbewegung in den 1970er Jahren hatte keine Auswirkungen auf die Vertretung von Frauen in Beschlussgremien der GTB. Solche Verhältnisse waren nicht auf die Gewerkschaften beschränkt; auch im Bundestag blieb es bis Ende der 1980er Jahre bei einer 90-Prozent-Männerquote. Selbst die Wahl von Monika Wulf-Mathies zur Vorsitzenden der großen Gewerkschaft Öffentliche Dienste, Transport und Verkehr (ÖTV) im Jahr 1982 löste in der »Frauengewerkschaft« GTB keine Revolution aus. Erst mit den Frauenförderplänen wurden ab 1986 Maßnahmen eingeleitet, die sowohl die Anzahl der Gewerkschaftssekretärinnen steigern als auch die Vertretung in allen Entscheidungsgremien geschlechtergerechter gestalten sollten (siehe Kapitel 5.4). Der Erfolg blieb zunächst beschränkt, bis die GTB mit der deutschen Wiedervereinigung deutlich weiblicher wurde. In mehreren ostdeutschen Verwaltungsstellen waren in allen Positionen ausschließlich Frauen tätig, die ihre Ansprüche deutlich artikulierten.

Dass die Frauen in der GTB die bisher abgelehnte harte Quote nach der Auflösung ihrer Gewerkschaft von der IG Metall bekamen, klingt fast wie eine Ironie am Ende der »Frauengewerkschaft«. In der 1998 beschlossenen Integrationsrichtlinie legte der Beirat der IG Metall fest, dass die Besetzung von Funktionen nach dem Anteil der Geschlechter an der Mitgliedschaft zu erfolgen hat (siehe Kapitel 8.1.1).

\subsection{Die Frauen im Geschäftsführenden Hauptvorstand}

In den fünfzig Jahren GTB-Geschichte gab es vier Vorsitzende und 21 weitere Mitglieder des Geschäftsführenden Hauptvorstandes (GHV), darunter nur drei Frauen (siehe Tabelle 1 im Anhang). Von 1963 bis 1969 gehörte keine einzige Frau dem GHV an; in den vierzig Jahren bis zur deutschen Wiedervereinigung schaffte es 
keine Frau auf die Position einer Bezirksleitung. Selbst in der Tarifabteilung des Hauptvorstandes war bis 1990 keine Frau in politischer Funktion tätig.

Was waren es für Frauen, die es unter diesen Bedingungen in das Führungsgremium geschafft hatten? Alle drei hatten einen proletarischen Hintergrund, begannen ihr Berufsleben in der Fabrik und behaupteten sich mit Engagement und Durchsetzungsvermögen in der GTB.

\subsubsection{Liesel Kipp-Kaule}

Im Jahr 1906 geboren, war Liesel Kipp-Kaule nach ihrer Schul- und Ausbildungszeit als Näherin in Bielefeld tätig. Sie wurde als geradezu bildungshungrig beschrieben, besuchte regelmäßig berufsbegleitende Kurse an der Volkshochschule und 1932 auch einen halbjährigen Kurs über Wirtschaftslehre, Geschichte und Politikwissenschaften in Thüringen. ${ }^{75}$ Schon in jungen Jahren schloss sie sich dem Deutschen Bekleidungsarbeiter-Verband an und wurde 1928 mit 22 Jahren zur Betriebsrätin gewählt, ein Amt, das sie bis 1933 wahrnehmen konnte. In der Weimarer Republik engagierte sie sich bei den Sozialdemokraten; nach der Machtergreifung der Nationalsozialisten besuchte sie von 1935 bis 1938 eine private Handelsschule in Bielefeld. Wegen kritischer Äußerungen wurde sie 1940 von der Gestapo verhört. Da sie seitdem beobachtet wurde, rieten ihr Freunde, die bisherige Arbeitsstelle zu verlassen. So verschlug es sie nach Offenbach, wo sie als kaufmännische Angestellte tätig war. ${ }^{76}$

Zurück in Ostwestfalen engagierte sie sich nach Kriegsende beim Aufbau der Gewerkschaft Textil-Bekleidung-Leder in Bielefeld und in der britischen Besatzungszone. Bereits im März 1946 wurde sie als politische Sekretärin eingestellt, beim Zonen-Gründungskongress ihrer Gewerkschaft im Jahr 1947 wurde sie in den Geschäftsführenden Vorstand gewählt. Im gleichen Jahr wählten die Delegierten sie auch als einziges weibliches Mitglied in den Vorstand des DGB für die britische Zone. Beim Gründungskongress des DGB für die Bundesrepublik Deutschland machten sich nicht nur die 14 weiblichen der 487 Delegierten für Liesel Kipp-Kaule als Mitglied des Geschäftsführenden Bundesvorstandes stark, bereits zuvor hatten viele Gewerkschaftsfrauen für ihre Wahl geworben. Auf dem Kongress selbst forderte Marta Sieger vom Freien Gewerkschaftsbund Hessen:

»Laßt uns selbst eine Frau vorschlagen, die wir uns als Leiterin in unseren Zusammenkünften, in unseren Ausschüssen ausgesucht haben. ${ }^{77}$

75 Notz (2003): Frauen in der Mannschaft, S. 285.

76 Notz (2003): Frauen in der Mannschaft, S. 286.

77 DGB: Protokoll des Gründungskongresses, 12.-14. Oktober 1949 in München, S. 236. 
Der designierte Vorsitzende Hans Böckler hatte jedoch der CDU/CSU die Zusage gemacht, dass zwei der sieben Vorstandspositionen aus der christlich-sozialen Arbeitnehmerbewegung kommen sollten. Mit der Wahl von Thea Harmuth, die CDU-Mitglied war, konnte man die informelle "Schwarzen-Quote« erfüllen und hatte zugleich eine Frau im Vorstand, so dass mit einer Person zwei Minderheiten berücksichtigt waren. ${ }^{78}$ Böcklers Autorität setzte sich schließlich durch und KippKaule erhielt beachtliche 197 Stimmen, Harmuth jedoch $276 .{ }^{79}$

Über die nordrhein-westfälische Landesliste der SPD war Liesel Kipp-Kaule zuvor in den Bundestag gewählt worden, dem sie bis 1965 angehörte. Bereits 1950 setzte sie sich gemeinsam mit ihrer Fraktionskollegin Lisa Albrecht für ein »Gesetz für ökonomische Gleichstellung der Frau« ein, das den Gleichheitsgrundsatz in Artikel 3 Grundgesetz ergänzen sollte, aber die beiden Politikerinnen konnten sich schon in der eigenen Partei nicht durchsetzen. Neben Gleichstellungsfragen engagierte sich Kipp-Kaule auch in der Sozialpolitik. So stritt sie im Bundestag leidenschaftlich für einen besseren Arbeitsschutz für werdende Mütter und für das Akkordverbot für junge Fabrikarbeiter:innen. ${ }^{80}$

Auch wegen der Arbeitsbelastung durch ihr Bundestagsmandat erhielt Liesel Kipp-Kaule im GHV mit der Frauenarbeit zunächst nur die Zuständigkeit für ein Themengebiet, später kam die Jugendarbeit dazu. Sie galt als resolute, durchsetzungsfähige Kollegin, was sich auch an der Art zeigte, wie sie die Gewerkschaftstage leitete. Dass sie im Hinblick auf die Frauenerwerbsarbeit ein »Kind ihrer Zeit« war, zeigt ihre Reaktion auf das Bedauern des damaligen Bundesfamilienministers Wuermeling, der Frauen wegen der steigenden Zahl der Ehescheidungen auf ihren »natürlichen Beruf«, Hausfrau und Mutter zu sein, zurückführen wollte. Kipp-Kaule hielt ihm auf dem Gewerkschaftstag 1957 entgegen, er solle sich darum kümmern,

»daß bessere Lohn- und Arbeitsbedingungen für unsere Männer geschaffen werden, damit unsere Frauen nicht aus wirtschaftlicher Not gezwungen werden, mitzuarbeiten ${ }^{81}$

Der Kongress solle den Auftrag geben,

78 Holland (2019): Gewerkschaftliche Geschlechterpolitik, S. 83.

79 DGB: Protokoll Gründungskongress, 12.-14. Oktober 1949 in München, S. 238.

80 Vgl. Meißner (2013): Erste Mutterschutzbestimmungen im Dt. Reich.

81 GTB: Protokoll des 5. Ordentlichen Gewerkschaftstages, 2.-5. Juli 1957 in Kassel, S. 120. 
»die Ansätze zu schaffen, daß wir in der Bundesrepublik Verhältnisse bekommen, die es ermöglichen, daß Frauen mit vorschulpflichtigen und schulpflichtigen Kindern aus wirtschaftlicher Not nicht gezwungen sind, zu arbeiten. $\ll^{82}$

Zum Gewerkschaftstag 1963 organisierte Liesel Kipp-Kaule erstmals ein bezirksübergreifendes Treffen der weiblichen Delegierten. In ihrem Geschäftsbericht betonte sie, dass in der GTB wohl alle verantwortlichen Gremien die Mitarbeit von Frauen für ausgesprochen wichtig hielten. Dennoch sollte der Gewerkschaftstag 1963 genau diese Aussage widerlegen: Liesel Kipp-Kaule unterlag bei den Wahlen zum GHV, bei denen es erstmals seit 1949 Kampfkandidaturen gab. Von den 232 Delegierten erhielt sie nur 111 Stimmen und lag damit 14 Stimmen hinter dem gewählten letzten Mitbewerber. Hildegard Tönjes, ehrenamtliches Mitglied des Hauptvorstandes, zeigte sich »aus tiefstem Herzen erschüttert«. Die Delegierte Gerda Grube bedauerte,

»daß nicht mehr Frauen als Delegierte hier sind, daß immer wieder Männer das Recht für sich in Anspruch nehmen, die Krone des Cewerkschaftstages zu sein und uns dann so in den Hintergrund stellen! ${ }^{83}$

Kipp-Kaule verabschiedete sich mit der Überzeugung, dass

»die Cleichberechtigung der Frau eine unerlässliche Voraussetzung für jede Demokratie ist. Die Bejahung der Demokratie als Staats- und Lebensform schließt selbstverständlich ein, dass man sich demokratischen Wahlentscheidungen unterwirft. $\ll^{84}$

1965 schied sie auch aus dem Bundestag aus. Liesel Kipp-Kaule verstarb 1992 in Bielefeld.

\subsubsection{Gerda Linde}

Die 1923 geborene Gerda Linde arbeitete in Essen als Weberin und engagierte sich schon in jungen Jahren im Betriebsrat. Bereits 1949, mit 28 Jahren, wurde sie Betriebsratsvorsitzende. 1954 wurde sie von ihrem Betrieb fristlos entlassen; erst über zwei Jahre später hob das Bundesarbeitsgericht die Kündigung auf. Diese

82 GTB: Protokoll des 5. Ordentlichen Gewerkschaftstages, 2.-5. Juli 1957 in Kassel, S. 121.

83 GTB: Protokoll des 8. Ordentlichen Gewerkschaftstages, 16.-20. September 1963 in Hannover, S. 328.

84 GTB: Protokoll des 8. Ordentlichen Cewerkschaftstages, 16.-20. September 1963 in Hannover, S. 326 . 
Auseinandersetzung durchzustehen war eine prägende Erfahrung für Gerda Linde. ${ }^{85}$

Früh übernahm sie auch Funktionen in der GTB, wurde von ihrer Verwaltungsstelle Essen für den Gewerkschaftstag 1953 als Delegierte gewählt und griff mit einem pointierten Beitrag in die Debatte zum Geschäftsbericht ein. Beim folgenden Kongress 1955 traf sie einen für die Organisation bisher ungewohnten emanzipatorischen Ton. Sie kritisierte die Frauenpolitik, die in keiner Weise systematisch betrieben werde. Man brauche die Förderung von Frauen, die aber nicht wieder »ein Lied mit 27 Strophen« werden dürfe. Linde initiierte einen Antrag, der verbindliche Richtlinien für die Frauenarbeit auf allen Ebenen der Organisation einforderte. Das meistens gönnerhafte Verhalten von Kollegen »unseren Frauen« gegenüber brachte sie folgendermaßen auf den Punkt:

»Wir Frauen machen den Männern den Vorwurf: Ihr unterdrückt uns. Ihr sagt: Im Gegenteil, wir mögen Euch so gern. Diese durchaus ernste Sache wird ins Lächerliche gezogen, wer leidet darunter? Unsere Organisation. ${ }^{86}$

Mit Beginn des Jahres 1957 wurde Gerda Linde bei der GTB eingestellt und begann im November ihre Tätigkeit als politische Sekretärin in der Bezirksleitung für Niedersachsen und Bremen. 1959 schlug die GTB Linde als Mitglied der Antragsberatungskommission für den DGB-Bundeskongress vor. Auch dort setzte sie sich mit dem Verhalten ihrer männlichen Kollegen auseinander. Zum Ende des von ihr vorgestellten Antragsblocks sagte sie:

»Es war augenfällig, daß in dem Moment, wo ich hier die Anträge zu bearbeiten hatte, der Kongress von einer Lebhaftigkeit befallen wurde, von der ich nicht hoffe, daß sie darauf zurückzuführen ist, daß unsere Kollegen, von denen wir ja zumindest in mancher Beziehung Kollegialität sicherlich immer wieder zu spüren bekommen, mich aus der Ruhe bringen wollten. ${ }^{87}$

Der Sitzungspräsident antwortete in genau dem Ton, den Linde schon Jahre zuvor auf dem GTB-Gewerkschaftstag bemängelt hatte, woraufhin das Protokoll Heiterkeit vermerkt:

85 Vgl. GTB: Protokoll des 15. Ordentlichen Cewerkschaftstages, 5.-9. Oktober 1986 in Aachen, S. 181.

86 CTB: Protokoll des 4. Ordentlichen Gewerkschaftstages, 5.-8. Juli 1955 in Frankfurt am Main, S. 129.

87 DCB: Protokoll des 5. Ordentlicher Bundeskongresses, 7.-12. September 1959 in Stuttgart, S. 218. 
»Ich darf der Kollegin Gerda Linde sagen, daß sie zweifellos auf den Kongress belebend gewirkt hat. ${ }^{88}$

Nach der Wahlniederlage von Liesel Kipp-Kaule wurde 1963 Martin Lange als neu gewähltem GHV-Mitglied die Zuständigkeit für die Frauenarbeit übertragen. Gerda Linde, nun politische Mitarbeiterin der Frauenabteilung beim Hauptvorstand, bekam dadurch eine besondere Rolle. Sie nahm als Gast an den Sitzungen des Hauptvorstandes teil - ein Zugeständnis, das die Kollegen als Reaktion auf die Abwahl Kipp-Kaules gemacht hatten. Nachdem Martin Lange 1969 während einer Sitzung des DGB-Bundesfrauenausschusses plötzlich verstorben war, wurde Linde vom Beirat als GHV-Mitglied nachgewählt und 1971 erstmals vom Gewerkschaftstag mit 166 von 209 Stimmen (79,4 Prozent) gewählt. Wie ihr Vorgänger war sie für Frauen- und Jugendpolitik und Mitgliederwerbung zuständig.

In Zusammenarbeit mit dem Zentralen Frauenausschuss, dem Spitzengremium der GTB-Frauenarbeit, erarbeitete Gerda Linde ein »Programm für die Arbeitnehmerin «, das vom Gewerkschaftstag 1971 beschlossen wurde. ${ }^{89}$ Bei der Einbringung des Antrags unterstrich sie als neues GHV-Mitglied, dass dies kein Alibiprogramm sei, sondern die GTB die Kraft der ganzen Organisation daransetze, die Forderungen durchzusetzen.

Schon auf ihrem ersten Gewerkschaftstag als GHV-Mitglied wurde deutlich, dass die stärker emanzipatorisch ausgerichtete Politik von Gerda Linde von ihren Kollegen im GHV argwöhnisch verfolgt wurde. Linde war die letzte im Führungsgremium verbliebene »Traditionalistin« oder »Linke« und machte zudem das gönnerhafte statt gleichberechtigte Verhalten von Kollegen immer wieder transparent. Dieses gespannte Verhältnis sollte bis zu ihrem altersbedingten Ausscheiden aus dem Amt bestehen bleiben. Ihre Kollegen lebten überwiegend in traditionellen Ehen mit dem heimischen »Ruhepol« der nicht berufstätigen Ehefrau. Lindes emanzipatorischer Ansatz nährte ihre Befürchtung des Machtverlusts, außerdem vertrat Gerda Linde in allgemeinpolitischen Fragen eher Positionen, wie sie von stärker linksgerichteten Gewerkschaften formuliert wurden. Die inhaltlichen Differenzen schlugen sich auch in Lindes Wahlergebnissen nieder: Sowohl auf dem Gewerkschaftstag 1978 als auch 1982 wurde sie mit dem jeweils niedrigsten Ergebnis wiedergewählt.

Als Frauenpolitikerin setzte Gerda Linde programmatisch Akzente. So betonte die GTB auf ihr Drängen hin in ihrem 1978 verabschiedeten Programm, dass Männer und Frauen gleichermaßen ein Recht auf Arbeit hätten. Berufstätigkeit, so heißt es dort, diene nicht nur der materiellen Existenzsicherung, sondern

88 DGB: Protokoll des 5. Ordentlichen Bundeskongresses, 7.-12. September 1959 in Stuttgart, S. 218.

89 GTB: Protokoll des 11. Ordentlichen Gewerkschaftstages, 4.-8. Oktober 1971 in Dortmund, S. $305 \mathrm{ff}$. 
auch der Persönlichkeitsentfaltung. ${ }^{90}$ Die Frauenausschüsse entwickelte Linde $\mathrm{zu}$ Arbeitskreisen für Frauenfragen, in denen nun auch Männer mitarbeiten sollten. Damit wurde eine Forderung erfüllt, die einzelne Kolleginnen seit den $1950 \mathrm{or}$ Jahren gestellt hatten: Frauenerwerbsarbeit und die damit verbundenen Probleme und Forderungen sollten gemeinsam diskutiert werden, um gemeinsam Verbesserungen erreichen zu können.

Bis zu ihrem Ausscheiden stritt Gerda Linde für die Selbstverständlichkeit von Frauenerwerbsarbeit und kämpfte gegen Vorurteile und ein antiquiertes Rollenbild, das sich ihrer Auffassung nach auch in der Politik der schwarz-gelben Bundesregierung seit 1983 widerspiegelte. Sie freute sich über das Selbstbewusstsein junger Frauen und unterstrich, dass Frauen auch im öffentlichen Leben nicht mehr sprachlos sein wollten. Frauen »brauchen keine Platzanweiser mehr, die ihnen ihre naturgegebene Rolle zuweisen ${ }^{91}{ }^{11}$ schrieb sie im Juli 1985 - Gleiches hatte sie mit anderen Worten schon dreißig Jahre zuvor auf dem Gewerkschaftstag gesagt.

Die Auseinandersetzung um allgemein- und frauenpolitische Streitfragen sowie die Jugendpolitik brachten Gerda Linde auch persönlich oft ans Limit. Nach wie vor ging sie keinem Streit mit ihren GHV-Kollegen aus dem Weg, was allerdings von der anderen Seite auch nicht behauptet werden kann. Anlässlich ihres Ausscheidens aus dem Hauptvorstand bescheinigte ihr der Vorsitzende Berthold Keller Leidenschaftlichkeit und fügte hinzu:

»Du hast im Ceschäftsführenden Hauptvorstand mit deiner Meinung nie zurückgehalten, auch dann nicht, wenn wir unterschiedlicher Meinung gewesen sind. Du hast für deine Auffassung gekämpft und gestritten. $\aleph^{92}$

Für ihren jahrzehntelangen Kampf um Gleichberechtigung erhielt Gerda Linde 1986 den Gustav-Heinemann-Bürgerpreis. Sie starb im Jahr 2016 in ihrer Heimatstadt Essen.

\subsubsection{Waltraud Hessedenz}

Die 1937 geborene Waltraud Hessedenz verschlug es nach dem Krieg nach Westdeutschland. Sie lernte in Herford den Beruf der Näherin in der Herrenoberbekleidung. Bald arbeitete sie als Springerin und engagierte sich in der Jugendarbeit der GTB. Ihre Fähigkeiten wurden in der Gewerkschaft eher erkannt als im Be-

90 GTB: Protokoll des 13. Ordentlichen Cewerkschaftstages, 1.-6. Oktober 1978 in Mannheim, S. 328.

91 Linde (1985): Frauen sind die Verlierer der Wende-Politik, S. 18.

92 GTB: Protokoll des 15. Ordentlichen Cewerkschaftstages, 5.-9. Oktober 1986 in Aachen, S. 181. 
trieb. So wurde sie vom damaligen ostwestfälischen Bezirksleiter Oskar Stieghorst ermuntert, zur Vorbereitung auf eine hauptamtliche Tätigkeit die Sozialakademie in Dortmund zu besuchen..$^{93}$ Anschließend wurde sie 1966 von der GTB angestellt und übernahm nach Ausbildungsstationen in Osnabrück und Bielefeld eine Stelle als politische Sekretärin bei der GTB in Bielefeld. Dort wurde sie 1971 zur Geschäftsführerin gewählt und leitete nun eine der größten Verwaltungsstellen der GTB, die vor allem wegen ihres Bekleidungsschwerpunktes mit hohem gewerkschaftlichen Organisationsgrad bundesweit bedeutend war.

Waltraud Hessedenz war nicht über die gewerkschaftliche Frauenarbeit sozialisiert worden und fremdelte mit dieser, wie sie später in einem Interview erklärte: »Mir ging es manchmal etwas zu gefühlsbetont zu. «" Sie war dadurch geprägt, dass sie ohne die Förderung von Kollegen nicht in diese Position gekommen wäre und wurde auch von Kolleginnen kritisiert, weil sie so früh Verantwortung in der Gewerkschaft übernommen hatte, statt sich als Mutter zurückzunehmen und ihren hauptamtlichen Kollegen den Vortritt zu lassen. Als Leiterin der mitgliederstärksten Bekleidungs-Verwaltungsstelle wollte sie sich nicht auf die Vertretung von Fraueninteressen beschränken und auf Frauenpolitik festlegen lassen. Wegen ihres großen Fachwissens genoss sie bei »ihren« Betriebsrät:innen und den Arbeitgebern hohe Anerkennung. Nach wenigen Jahren in der Bundestarifkommission ging kaum etwas gegen die Leiterin der Bielefelder Verwaltungsstelle.

In der Bielefelder Verwaltungsstelle wurde Mitgliedernähe großgeschrieben. Waltraud Hessedenz war überzeugt, dass der kurze Draht zu den Funktionär:innen zur Verbundenheit mit der Organisation führte. Jeder Betriebsrat stand zugleich für die Präsenz der GTB im Betrieb, was einen guten Organisationsgrad gewährleistete. Von Anfang an galt ihr Interesse auch der Sozialpolitik. Schon mit knapp 25 Jahren wurde sie auf Vorschlag ihres Bezirksleiters in die Vertreterversammlung der (Renten)Landesversicherungsanstalt Westfalen gewählt. Diese Aufgabe nahm sie 36 Jahre lang wahr.

Waltraud Hessedenz war Sozialdemokratin, stand politisch für eine konsequente Interessenvertretung und vertrat damit die Linie des vorstandskritischen ostwestfälischen Bezirks. Trotzdem kamen Berthold Keller und die Mehrheit im GHV an ihrer Person nicht vorbei, als es um die Nachfolge von Gerda Linde ging. So schlug Berthold Keller selbst Waltraud Hessedenz vor, die 1986 in den GHV gewählt wurde. Mit 160 von 209 Stimmen (76,6 Prozent) erzielte sie ein gutes Ergebnis; nur der Vorsitzende und zwei Kollegen erhielten mehr Stimmen.

93 Die Sozialakademie war eine wissenschaftliche Hochschule, die in der Nachkriegszeit gemeinsam vom Land Nordrhein-Westfalen, der Stadt Dortmund und dem DGB gegründet wurde, um Arbeitnehmer:innen zu qualifizieren und auf hauptamtliche sowie Leitungsfunktionen vorzubereiten.

94 Brücher/Buschmann/Link (2004): Starke Frauen, Arbeitskampf und Solidarität, S. 38 
Mit ihrer Praxiserfahrung brachte Waltraud Hessedenz neue Impulse für die Mitgliederwerbung. In der Jugendpolitik stand sie für Offenheit und Einbindung auch kritischer Kolleg:innen. In den 1980er Jahren lag das Augenmerk der Organisation und auch ihrer Kollegen im GHV auf der Frauenpolitik; der Gewerkschaftstag hatte den Auftrag zur Erstellung von Frauenförderplänen gegeben. Hessedenz betonte, dass es bei gewerkschaftlicher Frauenförderung nicht nur um die eigene Organisation gehen dürfe, sondern auch um die Bedingungen und Möglichkeiten für Frauen in den Betrieben - gerade hier sah sie die Aufgabe einer Gewerkschaft.

In der Öffentlichkeit wurde die verbindliche Frauenquote mittlerweile stärker diskutiert. Diese hätte in der GTB angesichts der Mitgliederstruktur weitreichende Konsequenzen gehabt - eine Befürchtung, die bei den GHV-Kollegen und Bezirksleitern deutlich mitschwang. Hessedenz' Erfahrungen im Führungsgremium der GTB unterschieden sich deutlich von ihrer vorherigen Arbeit in der Verwaltungsstelle. Dort war ihre Chefinnenrolle unumstritten und sie hatte $\mathrm{Au}$ torität, die auch in der Bundestarifkommission wirkte. Jetzt wurde jeder ihrer Schritte kritisch beäugt und im Umgang mit ihren Kollegen spürte sie Konkurrenzdenken und Misstrauen. Die Spitzenfunktionärinnen aus der Frauenarbeit waren ihre Stütze, auch der Zentrale Jugendausschuss stand hinter ihr, der aber weniger Einfluss hatte.

Beharrlich arbeitete Waltraud Hessedenz daran, die Frauenarbeit zu verbreitern und Konferenzen für Frauen und Jugendliche beteiligungsorientierter zu gestalten. Als es 1988 um den Einstieg in die 35-Stunden-Woche ging - eine Forderung, die gerade bei Frauen sehr populär war - konnte sie ihre Leidenschaft, die Tarifpolitik, mit der Frauenpolitik verbinden. Fortschritte waren eher in der Tarifals in der Gesellschaftspolitik zu erzielen, fiel doch die gesamte GHV-Amtszeit von Waltraud Hessedenz in die Regierungszeit von Bundeskanzler Helmut Kohl.

Im Ton verbindlicher als ihre Vorgängerin Gerda Linde, aber keineswegs weniger nachdrücklich, setzte sich Hessedenz für Fraueninteressen und -vertretung ein und hielt sich auch mit Kritik nicht zurück, wenn ihr die Entwicklung zu langsam ging. Nach der deutschen Wiedervereinigung litten Frauen wieder überproportional unter Arbeitslosigkeit. Die Krise, so Hessedenz; »belebt wieder ein Rollenbild von Frauen, das wir gerne zu den Akten gelegt hätten. ${ }^{95}$ Ihre politische Heimat blieb die SPD, insbesondere zur nordrhein-westfälischen Landesregierung hielt sie engen Kontakt. Von 1981 bis 1989 gehörte sie dem SPD-Landesvorstand Nordrhein-Westfalen an.

Als die GTB ab 1993 organisatorisch und finanziell unter Druck geriet, wurde der GHV verkleinert und Waltraud Hessedenz übernahm zusätzlich die Verantwortung für gewerkschaftliche Bildungsarbeit, Organisationspolitik und Beruf-

95 CTB: Ceschäftsbericht 1990-1993 des Hauptvorstandes, S. 169. 
liche Bildung. Mit anfangs geringer Überzeugung stimmte sie für den Weg der Integration in die IG Metall und musste 1997 ihre Funktion aus gesundheitlichen Gründen aufgeben.

Für ihr Engagement wurde Waltraud Hessedenz mit dem Bundesverdienstkreuz am Bande ausgezeichnet. 
